\title{
Reactive helicity and reactive power in nanoscale optics: Evanescent waves. Kerker conditions. Optical theorems and reactive dichroism
}

\author{
Manuel Nieto-Vesperinas $\odot^{1, *}$ and Xiaohao Xu $\odot^{2, \dagger}$ \\ ${ }^{1}$ Instituto de Ciencia de Materiales de Madrid, Consejo Superior de Investigaciones Científicas, \\ Campus de Cantoblanco, Madrid 28049, Spain \\ ${ }^{2}$ Institute of Nanophotonics, Jinan University, Guangzhou 511443, China
}

(Received 17 June 2021; accepted 6 October 2021; published 28 October 2021)

\begin{abstract}
Considering time-harmonic optical fields, we put forward the complex helicity and its alternating flow, together with their conservation equation: the complex helicity theorem. Its imaginary part constitutes a novel law that rules the build-up of what we establish as the reactive helicity through its zero time-average flow. Its associated reactive flow and the imaginary Poynting momentum that accounts for the accretion of reactive power are illustrated in two paradigmatic systems: evanescent waves and fields scattered from magnetodielectric dipolar nanoparticles. As for the former, we show that its reactive helicity may be experimentally observed as we introduce a reactive spin momentum and a reactive orbital momentum in terms of which we express the imaginary field momentum, whose transversal component produces an optical force on a magnetoelectric particle that, as we illustrate, may surpass and can be discriminated from the known force due to the so-called extraordinary momentum. We also uncover a nonconservative force on such a magnetoelectric particle, acting in the decay direction of the evanescent wave, and that may also be discriminated from the standard gradient force; thus making the reactive power of the wavefield also observable. Concerning the light scattered by magnetoelectric nanoparticles, we establish two optical theorems that govern the accretion of reactive helicity and reactive power on extinction of incident wave helicity and energy. Like a nule total, i.e., internal plus external, reactive power is at the root of a resonant scattered power, we show that a zero total reactive helicity underlies a resonant scattered helicity. These reactive quantities are shown to yield a novel interpretation of the two Kerker conditions, which we demonstrate to be linked to an absence, or minimum, of the overall scattered reactive energy. Further, we demonstrate that the first Kerker condition, under which the particle becomes dual on illumination with circularly polarized light, amounts to a nule overall scattered reactive helicity. Therefore these two reactive quantities are shown to underly the directivity of the particle scattering and emission. In addition, we discover a discriminatory property of the reactive helicity of chiral light incident on a chiral nanoparticle by excitation of the external reactive power. This should be useful for optical near field enantiomeric separation, an effect that we call reactive dichroism.
\end{abstract}

DOI: 10.1103/PhysRevResearch.3.043080

\section{INTRODUCTION}

Reactive quantities of electromagnetic fields, such as reactive and stored energy or the imaginary Poynting vector (IPV), are associated to physical entities that do not propagate in the environment, like evanescent waves in their varied forms as surface waves [1-3] or standing waves [3], along with near fields of RF antennas [3-10,12,13]. In recent years, advances in photonics and nano-optics led to the development of concepts such as chirality, helicity [14-20], magnetoelectric effects associated with the imaginary Poynting vector (IPV) [21], and its consequent transverse spin momen-

\footnotetext{
*mnieto@icmm.csic.es

†xuxhao_dakuren@163.com

Published by the American Physical Society under the terms of the Creative Commons Attribution 4.0 International license. Further distribution of this work must maintain attribution to the author(s) and the published article's title, journal citation, and DOI.
}

tum and magnetoelectric energy density [22-24], as well as related to the azimuthal imaginary Poynting momentum [25] and the Kerker-type nonconservative intensity gradient force [26].

In spite of progress in the analysis of optical antennas, mainly addressing quantum emitters and plasmonic nanoparticles [27-29], whose radiative and feeding characteristics are studied from the point of view of RF antennas and circuit theory [30-32], we have found few detailed studies [31] on their reactive quantities; although the effects of reactive power in antenna functionality are well-known [3,6-8,10$13,31]$; e.g., the radiative and reactive energies of an oscillating dipole are intertwined. In this way, a major task in RF-antenna design has been the study of its reactive power and $Q$ factor, seeking a minimization of both quantities in order to match the input energy with its radiative performance, since a large $Q$ and reactive energy in the antenna convey high ohmic losses, and a decrease of its operative bandwidth.

By contrast, at optical wavelengths, a high reactive power outside the nanoemitter or scatterer, external stored energy, 
and $Q$ factor of a low-loss nanoparticle, or nanoantenna, enhances both its scattered (or radiated) power and frequency sensitivity, also narrowing its operational bandwidth, this being desirable for its role as, e.g., a nanosource or a biosensor, as well as to reinforce light-matter interactions at the nanoscale, both in plasmonics [33] and Mie-tronics [34,35]. The reason is that, as we shall see, although such a large external reactive power and stored energy occur at wavelengths near those of resonant scattered power, the interior of the particle acts as a compensating (capacitive or inductive) element so that its reactive power and stored energy cancel out the external ones close to these resonant wavelengths at which each of these quantities have near extreme values. The result is that, in analogy with RF-antenna design [31], the total (i.e., internal plus external) reactive power and stored energy are zero close to resonances. We also show that the same effect occurs for the interior, external, and total reactive helicities in connection with a maximum efficiency in the helicity scattered up to the far zone.

Since surface plasmons work at optical frequencies where metals exhibit high losses, there has been an increasing interest in high refractive index nanoantennas, on which light exerts a magnetoelectric response with large electric and magnetic resonances [34-41]. We will study the key role of reactive quantities in the scattering from these magnetodielectric particles. The other archetypical configuration in which we shall address these reactive quantities is an evanescent wave.

Using time-harmonic wavefields, we will start addressing the flow of reactive power of the complex Poynting theorem, and since an analogous law for the helicity flow has never been established, as far as we know, we will introduce the concepts of complex helicity density [42] and complex helicity flow, for which we put forward a conservation equation that we coin as complex helicity theorem. Its real part is the well-known continuity equation for the conservation of optical helicity [14-17,19], while its imaginary part is a novel law that describes the build-up of reactive helicity, whose flow has zero time average and hence it does not propagate in free space.

We show that this reactive helicity density and its flow exist, like the reactive energy and the imaginary Poynting vector, in wavefields that do not propagate into the environment, as, e.g., evanescent and elliptically (and circularly in particular) polarized standing waves; the former being identical to the socalled magnetoelectric energy density, introduced in Ref. [24] following symmetry arguments but without providing its undelying physical law.

As for an evanescent wave, whose imaginary Poynting vector and its associated transversal spin have been studied [22], while its time-averaged energy flow, spin angular momentum, Belinfante momentum, and orbital momentum have to do with the time-averaged energy and helicity densities [22], we show that the reactive (i.e., imaginary) Poynting vector, and reactive helicity flow, are linked with the reactive energy and reactive helicity of the wave. These relationships provide a physical law for our introduced concept of reactive helicity, in which the aforementioned magnetoelectric energy [24], and the socalled "real helicity" discussed in a different research [43], are unified. Further, we discuss how the reactive power and reactive helicity of this wavefield are generated close to the interface through their corresponding alternate flows along the wave decay direction.

Moreover, we show that the imaginary Poynting momentum of the evanescent wavefield is the sum of densities of a reactive spin momentum and a reactive orbital momentum, which in turn are expressed as differences of the imaginary magnetic and electric corresponding momenta. This leads us to uncover a nonconservative optical force on a magnetodielectric particle, directed along the wave decay direction-i.e., different from the gradient force and larger than it at certain wavelengths, thus being experimentally detectable-due to the IPV.

We also illustrate the presence of a transversal force on such a magnetodielectric particle, stemming from the corresponding component of the IPV, which may be of opposite sign and much larger than the known lateral force [22] due to the time-averaged Poynting vector; thus being detectable and making observable the reactive helicity.

As regards fields emitted or scattered by a magnetodielectric dipolar nanoparticle, we shall show that its reactive energy and angular distribution of scattered radiation, being intimately interrelated, provide a novel interpretation of the two Kerker conditions: K1 and K2 [37,44-49], and hence of the particle emission directivity. Namely, under plane-wave illumination, and at wavelengths where such a particle fulfils either $\mathrm{K} 1$ of zero backscattering, or $\mathrm{K} 2$ of minimum forward scattering, the overall external reactive power around this body is either zero or almost zero, while the internal and total (i.e., external plus internal) reactive powers are near zero. Moreover, the overall external reactive helicity vanishes at $\mathrm{K} 1$ wavelengths on illumination with circularly polarized light.

Concerning feeding the magnetodielectric nanoantenna, we put forward the reactive power and the reactive helicity optical theorems, which quantify the accretion of external stored reactive energy and reactive helicity in the near and intermediate field zones, in terms of the particle excitation and extinction of energy and helicity of the supplied illumination. The effects here shown maximizing both the external and internal values of these reactive quantities, (while minimizing their overall amounts, i.e., external plus internal), which tune to resonance their radiation efficiency, establish an analogy with the same well-known pursuit in RF antenna design. Therefore this work puts forward the importance of reactive quantities which underly some previously studied concepts in the analysis of optical antennas [27-30].

Finally, we show that the reactive power theorem yields an interpretation of how by illuminating a chiral particle with a nonfree propagating wavefield, like an elliptically polarized standing wave, evanescent wave, or near field from a nearby emitter, the reactive helicity of the incident wavefield appears as a consequence of the generation of reactive energy on interaction with the sample particle. It is intriguing that, as we find, this incident reactive helicity emerges analogously as the incident helicity does in a standard dichroism far field observation [14]. Therefore we show that the socalled "magnetoelectric response" of a chiral particle, quoted in Ref. [24], arises as a consequence of the accretion of its reactive energy from near field chiral illumination, e.g., with incident evanescent waves (or, similarly, with incident circularly polarized standing waves as proposed in Ref. [24]). 
As such, we name this magnetoelectric phenomenon reactive dichroism . It underlines the observability of such incident reactive helicity, and its discriminatory property for enantiomer separation by near field optical techniques using structured illumination.

\section{THE REACTIVE POYNTING VECTOR AND THE REACTIVE POWER}

To fix some concepts to deal with, we first outline the main quantities involved in the complex Poynting theorem. We shall assume an arbitrary body immersed in a lossless homogeneous medium [50]. Let $\mathcal{E}(\mathbf{r}, t)=\mathbf{E}(\mathbf{r}) \exp (-i \omega t)$, $\mathcal{B}(\mathbf{r}, t)=\mathbf{B}(\mathbf{r}) \exp (-i \omega t)$ be a time-harmonic electromagnetic field. It is well-known that in a body with charges and free electric currents of density $\mathbf{j}$ contained in a volume $V$ with permitivity $\epsilon$ and permeability $\mu$, the complex work density, given by the scalar product $\mathbf{j}^{*} \cdot \mathbf{E}$, leads after using Maxwell's equations, $\nabla \times \mathcal{E}=$ $-(1 / c) \partial \mathcal{B} / \partial t, \quad \nabla \times \mathcal{H}=(4 \pi / c) \mathcal{J}+(1 / c) \partial \mathcal{D} / \partial t,[\mathcal{D}=$ $\epsilon \mathcal{E}, \mathcal{B}=\mu \mathcal{H}, \mathcal{J}(\mathbf{r}, t)=\mathbf{j}(\mathbf{r}) \exp (-i \omega t)]$, to the complex Poynting theorem $[3,5,9,12]$ :

$$
\begin{aligned}
\int_{V}\left[\frac{1}{2} \mathbf{j}^{*} \cdot \mathbf{E}+\nabla \cdot \mathbf{S}\right] d^{3} r & =i 2 \omega \int_{V}\left(\left\langle w_{m}\right\rangle-\left\langle w_{e}\right\rangle\right) d^{3} r \\
\omega & =\frac{k c}{\sqrt{\epsilon \mu}}=\frac{2 \pi}{\lambda} \frac{c}{\sqrt{\epsilon \mu}} .
\end{aligned}
$$

Where $*$ and $\langle$.$\rangle mean complex conjugated and time-average,$ respectively. The complex Poynting vector (CPV) S, and timeaveraged electric and magnetic energy densities, $\left\langle w_{e}\right\rangle$ and $\left\langle w_{m}\right\rangle$, are

$$
\begin{aligned}
\mathbf{S}(\mathbf{r}) & =\frac{c}{8 \pi \mu} \mathbf{E}(\mathbf{r}) \times \mathbf{B}^{*}(\mathbf{r}), \\
\left\langle w_{e}(\mathbf{r})\right\rangle & =\frac{\epsilon}{16 \pi}|\mathbf{E}(\mathbf{r})|^{2}, \quad\left\langle w_{m}(\mathbf{r})\right\rangle=\frac{1}{16 \pi \mu}|\mathbf{B}(\mathbf{r})|^{2} .
\end{aligned}
$$

Under our above assumptions, the right-hand side of (1) is purely imaginary, and the real part of this equation constitutes the well-known Poynting theorem describing the variation of energy in the body due to the work rate of the field upon its charges, given by the volume integral of $\frac{1}{2} \operatorname{Re}\left\{\mathbf{j}^{*}\right.$. $\mathbf{E}\}$. This variation is characterized by the flow of the timeaveraged Poynting vector, $\langle\mathbf{S}\rangle=\operatorname{Re}\{\mathbf{S}\}$, across the surface $\partial V$ of $V: \int_{\partial V} \operatorname{Re}\{\mathbf{S}\} \cdot \hat{\mathbf{r}} d^{2} r$, where $\hat{\mathbf{r}}$ is the outward unit normal to $\partial V$.

On the other hand, the imaginary part of (1) formulates that in the steady state the integral of the imaginary work $\frac{1}{2} \operatorname{Im}\left\{\mathbf{j}^{*} \cdot \mathbf{E}\right\}$, plus the reactive power flux $\int_{\partial V} \operatorname{Im}\{\mathbf{S}\} \cdot \mathbf{n} d^{2} r$ (which has zero time average since it is associated with an instantaneous energy flow that alternates back and forth at twice the frequency across $\partial V$ ) accounts for this reactive power build-up in and around the body, given by the righthand side of $(1) ; 2\left(\left\langle w_{m}\right\rangle-\left\langle w_{e}\right\rangle\right)$ being the reactive energy density. Unless otherwise stated, we shall henceforth assume $\epsilon=\mu=1$ for the embedding space.

At this point, it is convenient to introduce the instantaneous Poynting vector [3,12] built by the fields $\mathcal{E}(\mathbf{r}, t)=\mathfrak{R}\{\mathcal{E}(\mathbf{r}, t)\}$ and $\mathcal{B}(\mathbf{r}, t)=\mathfrak{R}\{\mathcal{B}(\mathbf{r}, t)\}$,

$$
\begin{aligned}
\mathcal{S}(\mathbf{r}, t) & =\frac{c}{4 \pi} \mathcal{E}(\mathbf{r}, t) \times \mathcal{B}(\mathbf{r}, t) \\
& =\langle\mathbf{S}\rangle+\frac{c}{8 \pi} \operatorname{Re}\{\mathbf{E}(\mathbf{r}) \times \mathbf{B}(\mathbf{r}) \exp (-2 i \omega t)\} .
\end{aligned}
$$

This is the standard expression of $\mathcal{S}(\mathbf{r}, t)$. However, we find it more instructive to write it as

$$
\begin{aligned}
\mathcal{S}(\mathbf{r}, t)= & \langle\mathbf{S}\rangle(1+\cos 2 \omega t)+\operatorname{Im}\{\mathbf{S}\} \sin 2 \omega t \\
& +\frac{c}{4 \pi} \mathbf{E}^{R}(\mathbf{r}) \times \mathbf{B}^{I}(\mathbf{r}) \sin 2 \omega t \\
& -\frac{c}{4 \pi} \mathbf{E}^{I}(\mathbf{r}) \times \mathbf{B}^{I}(\mathbf{r}) \cos 2 \omega t,
\end{aligned}
$$

where the superscripts $R$ and $I$ denote real and imaginary parts, respectively. Note that while the term with $\langle\mathbf{S}\rangle$ does not change sign, as expected from that part of the instantaneous Poynting vector associated with the time-averaged energy flow, the term that contains the IPV or reactive Poynting vector, $\operatorname{Im}\{\mathbf{S}\}$, alternates its sign at frequency $2 \omega$ following the variation of $\sin 2 \omega t$. This is in accordance with the above interpretation of the imaginary part of (1). We also see that there is a generally nonzero contribution to this alternating flow in the last two terms of (3). Obviously, only the $\langle\mathbf{S}\rangle$ term remains on time averaging in (3).

\section{The reactive Poynting vector and the angular spectrum of plane waves}

To distinguish the structure of the real and imaginary parts of $\mathbf{S}$, it helps to employ the angular spectrum representation of the electromagnetic field, so as to map these quantities into their spectra in Fourier space. To this end, we calculate the flow of $\frac{c}{8 \pi} \mathbf{E}(\mathbf{r}) \times \mathbf{B}^{*}(\mathbf{r})$ across a plane $z=$ constant. We use for simplicity a framework such that the sources are on $z<0$ and thus the integration is done on the $z=0$ plane. But one may equally choose any other constant value $z=z_{0}$, providing the sources lie in $z<z_{0}$. The electric field $\mathbf{E}(\mathbf{r})$ propagating into the half-space $z \geqslant 0$ is represented by its angular spectrum of plane-wave components as [51,52]

$$
\mathbf{E}(\mathbf{r})=\int_{-\infty}^{\infty} d^{2} \mathbf{K e}(\mathbf{K}) \exp \left[i\left(\mathbf{K} \cdot \mathbf{R}+k_{z} z\right)\right]
$$

$\mathbf{r}=(\mathbf{R}, z), \mathbf{R}=(x, y)$,

$$
\begin{aligned}
\mathbf{k}= & \left(\mathbf{K}, k_{z}\right), \mathbf{K}=\left(K_{x}, K_{y}\right),|\mathbf{k}|^{2}=k^{2} ; \text { and } \\
k_{z}= & \sqrt{k^{2}-K^{2}}=q_{h}, K \leqslant k, \text { for homogeneous } \\
& \text { (propagating) plane-wave components. } \\
k_{z}= & i \sqrt{K^{2}-k^{2}}=i q_{e}, K>k, \text { for evanescent } \\
& \text { plane-wave components. }
\end{aligned}
$$

Using the subscripts $h$ and $e$ for homogeneous and evanescent components, respectively, the CPV flux, $\Phi^{\text {Poynt }}$, across the plane $z=0$ has real and imaginary parts given by (see the proof in Appendix A)

$$
\Phi^{\text {RPoynt }}=\frac{\pi c}{2 k} \int_{K \leqslant k} d^{2} \mathbf{K} q_{h}\left|\mathbf{e}_{h}(\mathbf{K})\right|^{2}
$$


and

$$
\Phi^{\mathrm{IPoynt}}=-\frac{\pi c}{2 k} \int_{K>k} d^{2} \mathbf{K}\left[q_{e}\left|\mathbf{e}_{e}(\mathbf{K})\right|^{2}-2 q_{e}\left|e_{e z}(\mathbf{K})\right|^{2}\right] .
$$

Equation (6) is well-known, it expresses the flux $\Phi^{\text {RPoynt }}$ of the real part of the CPV as the momentum power carried on by the propagating components $(K \leqslant k)$. However, Eq. (7) shows that the flux $\Phi^{\text {IPoynt }}$ of the reactive CPV is momentum associated with power contained in the evanescent components $(K>k)$, and as such it does not propagate into $z \geqslant 0$. Notice the special role played by the longitudinal component $e_{e z}(\mathbf{K})$ in (7).

\section{THE REACTIVE HELICITY AND ITS REACTIVE FLOW THEOREM}

The optical helicity density of the electromagnetic field in a medium with constitutive parameters $\epsilon$ and $\mu$ is well-known to be $[15,17,19]$

$$
\mathscr{H}(\mathbf{r})=(1 / 2 k) \sqrt{\frac{\epsilon}{\mu}} \operatorname{Im}\left\{\mathbf{E}(\mathbf{r}) \cdot \mathbf{B}^{*}(\mathbf{r})\right\} .
$$

We now introduce the quantity

$$
\mathscr{H}_{\mathscr{R}}(\mathbf{r})=(1 / 2 k) \sqrt{\frac{\epsilon}{\mu}} \operatorname{Re}\left\{\mathbf{E}(\mathbf{r}) \cdot \mathbf{B}^{*}(\mathbf{r})\right\} .
$$

Like $\operatorname{Im}\{\mathbf{S}\}$, this quantity $\mathscr{H}_{\mathscr{R}}$ may appear from $\mathscr{H}$ when $\mathbf{E}$ and $\mathbf{B}$ are chosen $\pi / 2$ out of phase.

We shall later see that $\mathscr{H}_{\mathscr{R}}$ is exclusive of $\operatorname{Im}\{\mathbf{S}\}$ (and, of course, of a reactive helicity flow, to be introduced next). Thus we call $\mathscr{H}_{\mathscr{R}}$ the reactive helicity density of the field. Some authors have recently addressed this quantity in different works, and call it magnetoelectric energy [24], or just real helicity [43]. However, we keep our denomination by showing that, like the reactive energy, it fulfills a conservation law.

We consider a body with charges and free currents, embedded in a volume $V$. From the two Maxwell equations for the spatial vectors, $\nabla \times \mathbf{E}=i k \mathbf{B}$ and $\boldsymbol{\nabla} \times \mathbf{H}=(4 \pi / c) \mathbf{j}-i k \mathbf{D}$, we derive the conservation equation for the reactive helicity. First, we employ the second of these equations and address the scalar product $(4 \pi / c) \mathbf{j}^{*} \cdot \mathbf{B}$ in $V$. On using the identity $\mathbf{B} \cdot\left(\nabla \times \mathbf{H}^{*}\right)=\mathbf{H}^{*} \cdot(\nabla \times \mathbf{B})-\nabla \cdot\left(\mathbf{B} \times \mathbf{H}^{*}\right)$, one gets

$$
\int_{V}\left[-\frac{2 \pi}{k n} \operatorname{Im}\left\{\mathbf{j}^{*} \cdot \mathbf{B}\right\}+\nabla \cdot \mathcal{F}_{B}\right] d^{3} r=\omega \int_{V} d^{3} r \mathscr{H}_{\mathscr{R}} .
$$

In (10), $\mathcal{F}_{B}=(c / 4 k n) \Im\left\{\mathbf{H}^{*} \times \mathbf{B}\right\}$ is the density of magnetic flow of helicity $[15,17,24](n=\sqrt{\epsilon \mu})$; and it is also proportional to the magnetic part of the spin angular momentum density [15,17,24] [see also Eq. (C2) below].

Similarly, we may obtain a conservation equation for $\mathscr{H}_{\mathscr{R}}$ with the electric helicity flow density, $\mathcal{F}_{E}=(c / 4 k n) \operatorname{Im}\left\{\mathbf{E}^{*} \times\right.$ D . This is done by taking the scalar product: $\mathbf{D} \cdot\left(\nabla \times \mathbf{E}^{*}\right)=$ $-i k \mathbf{D} \cdot \mathbf{B}^{*}$, and proceeding in an identical way as with the derivation of (10), the result is

$$
\int_{V} \nabla \cdot \mathcal{F}_{E} d^{3} r=-\omega \int_{V} \mathscr{H}_{\mathscr{R}} d^{3} r
$$

Adding (11) and (10) one obtains the well-known continuity equation for the conservation of helicity in the steady state:

$$
\int_{V} \nabla \cdot \mathcal{F} d^{3} r \equiv \int_{\partial V} \mathcal{F} \cdot \hat{\mathbf{r}} d^{2} r=\frac{2 \pi}{k n} \int_{V} \operatorname{Im}\left\{\mathbf{j}^{*} \cdot \mathbf{B}\right\} d^{3} r,
$$

which shows that the flow of helicity, or dual-symmetric spin [17,53],

$$
\mathcal{F}=\mathcal{F}_{E}+\mathcal{F}_{B}=(c / 4 k n) \operatorname{Im}\left\{\mathbf{E}^{*} \times \mathbf{D}+\mathbf{H}^{*} \times \mathbf{B}\right\},
$$

across the boundary $\partial V$ of $V$ equals the radiated field helicity, including its dissipation and conversion given by the righthand side of (12) [54-56].

However, subtracting (11) from (10) leads to the reactive helicity flow theorem,

$$
\int_{V}\left[-\frac{2 \pi}{k n} \operatorname{Im}\left\{\mathbf{j}^{*} \cdot \mathbf{B}\right\}+\nabla \cdot \mathcal{F}_{\mathscr{H}_{\mathscr{R}}}\right] d^{3} r=2 \omega \int_{V} \mathscr{H}_{\mathscr{R}} d^{3} r
$$

where we have introduced the reactive helicity flow associated with a flow of helicity that vanishes on time average, although not instantly,

$$
\begin{aligned}
\mathcal{F}_{\mathscr{H}_{\mathscr{R}}} & =\mathcal{F}_{B}-\mathcal{F}_{E}=(c / 4 k n) \mathfrak{\Im}\left\{\mathbf{H}^{*} \times \mathbf{B}-\mathbf{E}^{*} \times \mathbf{D}\right\} \\
& =\frac{2 \pi c^{2}}{n}\left(\left\langle\mathcal{S}_{m}\right\rangle-\left\langle\mathcal{S}_{e}\right\rangle\right) .
\end{aligned}
$$

If $\partial V$ is in air or vacuum, $n=1, \mathbf{B}=\mathbf{H}$, and $\mathbf{D}=\mathbf{E}$ in (13) and (15). $\left\langle\mathcal{S}_{e}\right\rangle$ and $\left\langle\mathcal{S}_{m}\right\rangle$ stand for the time averages of the density of electric and magnetic spin angular momentum, (cf. Appendix C).

The quantity $2 \omega \mathscr{H}_{\mathscr{R}}$ is a reactive helicity per unit halfperiod, or just the reactive helicity power, in analogy with the reactive power of the complex Poynting theorem, Eq. (1). Hence (14) expresses the conservation of $2 \omega \mathscr{H}_{\mathscr{R}}$. Indeed, (12) and (14) suggest us to formulate a complex helicity theorem [57]:

$$
\begin{aligned}
\int_{V}\left[-(1+i) \frac{2 \pi}{k n} \operatorname{Im}\left\{\mathbf{j}^{*} \cdot \mathbf{B}\right\}+\nabla \cdot \mathcal{F}_{C}\right] d^{3} r & =2 i \omega \int_{V} \mathscr{H}_{\mathscr{R}} d^{3} r, \\
\mathcal{F}_{C} & =\mathcal{F}+i \mathcal{F}_{\mathscr{H}_{\mathscr{R}}}
\end{aligned}
$$

where the complex helicity flow, or complex spin angular momentum, is $\mathcal{F}_{C}$. Evidently, (16) has a real part, which is the standard helicity conservation equation (12), whereas its imaginary part is Eq. (14) for the reactive helicity flow and governs the variation of reactive helicity in $V$, given by the decrease of the integrated source density $-\frac{2 \pi}{k n} \operatorname{Im}\left\{\mathbf{j}^{*} \cdot \mathbf{B}\right\}$. This variation is expressed in terms of the reactive helicity flux $\int_{\partial V} \mathcal{F}_{\mathscr{H}_{\mathscr{R}}} \cdot \hat{\mathbf{r}} d^{2} r$ (which has zero time average since it comes from $\Im\left\{\mathcal{F}_{C}\right\}$, and hence represents helicity flowing back and forth to the body across $\partial V$ in its near field region, without net propagation), and of integrated reactive helicity density $2 \omega \mathscr{H}_{\mathscr{R}}$.

Unless otherwise stated, we shall not drag the factor $2 \omega$ when we refer to the reactive helicity, thus we shall just write $\mathscr{H}_{\mathscr{R}}$ for this quantity. In Sec. VII, we show that $\mathscr{H}_{\mathscr{R}}$ is built-up on chiral light-matter interaction. This gives rise to the phenomenon of reactive dichroism in the near field of the body, addressed in Sec. VIII. 
Equations (10) and (11) suggest that $\mathscr{H}_{\mathscr{R}}$ is observable, for example, by detecting the torque exerted by a circularly polarized plane wave on a dipolar particle on which the field induces a purely electric (e) or a purely magnetic (m) dipole. In fact, in vacuum, $\mathcal{F}_{E}$ and $\mathcal{F}_{B}$ are proportional to the optical electric and magnetic torque, respectively, $\boldsymbol{\Gamma}_{E}=(1 / 8 \pi k) \sigma_{e}^{(a)} \operatorname{Im}\left\{\mathbf{E}^{*} \times \mathbf{E}\right\}=c \sigma_{e}^{(a)}\left\langle\mathcal{S}_{e}\right\rangle$ and $\boldsymbol{\Gamma}_{B}=(1 / 8 \pi k) \sigma_{m}^{(a)} \operatorname{Im}\left\{\mathbf{B}^{*} \times \mathbf{B}\right\}=c \sigma_{m}^{(a)}\left\langle\mathcal{S}_{m}\right\rangle ; \sigma_{m}^{(a)}$ and $\sigma_{m}^{(a)}$ being the particle electric and magnetic absorption cross sections, (see Ref. [58], Sec. X). Otherwise, if the particle is magnetodielectric, $\mathscr{H}_{\mathscr{R}}$ becomes observable through the e-m interaction force, see Eq. (31) below, [cf. Ref. [21] Eq. (44)]. This latter situation is detailed with an evanescent wave in Sec. IV B, cf. Eq. (23).

Concerning the $P, T$, and $D$ symmetries of these novel quantities, namely,

parity, $P: \mathbf{r} \rightarrow-\mathbf{r}, P: \mathbf{E} \rightarrow-\mathbf{E}, P: \mathbf{B} \rightarrow \mathbf{B}$;

time-reversal, $T: t \rightarrow-t, T: \mathbf{E} \rightarrow \mathbf{E}^{*}, T: \mathbf{B} \rightarrow-\mathbf{B}^{*}$;

and duality, $D: \mathbf{E} \rightarrow \mathbf{B}, D: \mathbf{B} \rightarrow-\mathbf{E}$,

while it is well-known that

$$
\begin{aligned}
& P:\langle\mathbf{S}\rangle \rightarrow-\langle\mathbf{S}\rangle, P: \mathcal{F} \rightarrow \mathcal{F}, P: \mathscr{H} \rightarrow-\mathscr{H} ; \\
& T:\langle\mathbf{S}\rangle \rightarrow-\langle\mathbf{S}\rangle, T: \mathcal{F} \rightarrow-\mathcal{F}, T: \mathscr{H} \rightarrow \mathscr{H} ; \\
& D:\langle\mathbf{S}\rangle \rightarrow\langle\mathbf{S}\rangle, D: \mathcal{F} \rightarrow \mathcal{F}, D: \mathscr{H} \rightarrow \mathscr{H} ;
\end{aligned}
$$

and we obtain the symmetries for the reactive quantities:

$$
\begin{aligned}
& P: \operatorname{Im}\{\mathbf{S}\} \rightarrow-\operatorname{Im}\{\mathbf{S}\}, P: \mathcal{F}_{\mathscr{H}_{\mathscr{R}}} \rightarrow \mathcal{F}_{\mathscr{H}_{\mathscr{R}}}, \\
& P: \mathscr{H}_{\mathscr{R}} \rightarrow-\mathscr{H}_{\mathscr{R}} \\
& T: \operatorname{Im}\{\mathbf{S}\} \rightarrow \operatorname{Im}\{\mathbf{S}\}, T: \mathcal{F}_{\mathscr{H}_{\mathscr{R}}} \rightarrow-\mathcal{F}_{\mathscr{H}_{\mathscr{R}}}, \\
& T: \mathscr{H}_{\mathscr{R}} \rightarrow-\mathscr{H}_{\mathscr{R}} ; \\
& D: \operatorname{Im}\{\mathbf{S}\} \rightarrow-\operatorname{Im}\{\mathbf{S}\}, D: \mathcal{F}_{\mathscr{H}_{\mathscr{R}}} \rightarrow-\mathcal{F}_{\mathscr{H}_{\mathscr{R}}}, \\
& D: \mathscr{H}_{\mathscr{R}} \rightarrow-\mathscr{H}_{\mathscr{R}}
\end{aligned}
$$

It is interesting that while under parity the three reactive quantities behave like their corresponding nonreactive counterparts, they invert their symmetry under duality applications, in contrast with their nonreactive analogues that remain $D$-invariant. Under time-reversal, only $\mathcal{F}_{\mathscr{H}_{\mathscr{R}}}$ has the same symmetry as its nonreactive analogue $\mathcal{F}$.

\section{The reactive helicity and the angular spectrum}

In order to gain more insight into the different nature of $\mathscr{H}$ and $\mathscr{H}_{\mathscr{R}}$, we shall employ once again the angular spectrum of the electromagnetic wave. We evaluate the total $\mathscr{H}$ and $\mathscr{H}_{\mathscr{R}}$ per unit $z$ length by integration of $\mathbf{E}(\mathbf{r}) \cdot \mathbf{B}^{*}(\mathbf{r})$ on a plane $z=$ constant. Again, we choose coordinates such that the sources are on $z<0$ and thus the integration is done on the $z=0$ plane.

Using, as before, the subindex $h$ and $e$ for homogeneous and evanescent components, respectively, the integral on $z=$ 0 of $\mathbf{E}(\mathbf{r}) \cdot \mathbf{B}^{*}(\mathbf{r})$ is shown in Appendix B to yield

$$
\begin{aligned}
\int_{-\infty}^{\infty} d^{2} \mathbf{R} \mathscr{H}(\mathbf{R}, 0)= & (1 / 2 k) \sqrt{\frac{\epsilon}{\mu}} \int_{-\infty}^{\infty} d^{2} \mathbf{R} \operatorname{Im}\left\{\mathbf{E}(\mathbf{R}) \cdot \mathbf{B}^{*}(\mathbf{R})\right\} \\
= & 2 \frac{(2 \pi)^{2}}{k c}\left\{\int_{K \leqslant k} d^{2} \mathbf{K k}_{h} \cdot \mathcal{F}_{E h}(\mathbf{K})\right. \\
& \left.+\int_{K>k} d^{2} \mathbf{K} \mathbf{K} \cdot \mathcal{F}_{E e \perp}(\mathbf{K})\right\}
\end{aligned}
$$

and

$$
\begin{aligned}
\int_{-\infty}^{\infty} d^{2} \mathbf{R} \mathscr{H}_{\mathscr{R}}(\mathbf{R}, 0) & =(1 / 2 k) \sqrt{\frac{\epsilon}{\mu}} \int_{-\infty}^{\infty} d^{2} \mathbf{R} \operatorname{Re}\left\{\mathbf{E}(\mathbf{R}) \cdot \mathbf{B}^{*}(\mathbf{R})\right\} \\
& =2 \frac{(2 \pi)^{2}}{k c} \int_{K>k} d^{2} \mathbf{K} q_{e} \mathcal{F}_{E e z}(\mathbf{K}) .
\end{aligned}
$$

In (17) $\mathcal{F}_{E e}=\left(\mathcal{F}_{E e \perp}, \mathcal{F}_{E e z}\right), \mathcal{F}_{E e \perp}=\left(\mathcal{F}_{E e x}, \mathcal{F}_{E e y}\right)$. Therefore Eq. (17) shows that in the domain of propagating components the helicity density of the field in $z=0$ maps onto the projection of the electric spin angular momentum of the K-plane-wave component onto the propagation wave vectors; namely, onto the real wave vector $\mathbf{k}_{h}$, while in the evanescent region, it is given by the projection of the electric spin onto the transversal (propagating) component $\mathbf{K}$. (Note that this is in agreement with the standard definition [16], but here it is generalized to include evanescent waves.)

Of special interest is, however, Eq. (18), which shows that the reactive helicity density $\mathscr{H}_{\mathscr{R}}$ maps in $\mathbf{K}$ space as the projection of the electric spin of the Kth-evanescent component onto the $z$ component $i \mathbf{k}_{z}^{*}=i\left(-i q_{e}\right) \hat{z}$. Where $\mathbf{k}$ is the complex wave vector of this Kth-evanescent wave [cf. Eq. (5)]. Again, this justifies that we call reactive the real part of $\mathbf{E}(\mathbf{r}) \cdot \mathbf{B}^{*}(\mathbf{r})$.

In this connection, it should be remarked that like the reactive power and the IPV are linked with nonpropagating waves, e.g., evanescent and standing waves [3], the reactive helicity $\mathscr{H}_{\mathscr{R}}$ (and hence its flow $\mathcal{F}_{\mathscr{H}_{\mathscr{R}}}$ ) exist in evanescent waves as shown in Eq. (18), as well as in elliptically polarized (and circularly polarized in particular, CPL) standing wavefields [59]. For CPL waves the authors of [24] used the term "magnetoelectric energy" which, as seen above, is a reactive quantity since it is the same as $\mathscr{H}_{\mathscr{R}}$.

\section{CASE 1: REACTIVE POWER, REACTIVE HELICITY, AND REACTIVE MOMENTA IN AN EVANESCENT WAVE}

As illustrated in Fig. 1, we consider a generic timeharmonic evanescent wave in air, $z \geqslant 0$, generated by total internal reflection (TIR), at a plane interface $z=0$ separating air $(\epsilon=\mu=1)$ from a dielectric in the half-space $z \leqslant 0$. The plane of incidence being $O X Z$. Then the complex spatial parts of the electric and magnetic vectors in $z>0$ are expressed in a Cartesian coordinate basis $\{\hat{\mathbf{x}}, \hat{\mathbf{y}}, \hat{\mathbf{z}}\}$ as $(n=1)[60,61]$ :

$$
\begin{aligned}
& \mathbf{E}=\left(-\frac{i q}{k} T_{\|}, T_{\perp}, \frac{K}{k} T_{\|}\right) \exp (i K x-q z), \\
& \mathbf{B}=n\left(-\frac{i q}{k} T_{\perp},-T_{\|}, \frac{K}{k} T_{\perp}\right) \exp (i K x-q z) .
\end{aligned}
$$

For TE or $s$ (TM or $p$ ) polarization, i.e., $\mathbf{E}^{(i)}\left(\mathbf{B}^{(i)}\right)$ perpendicular to the plane of incidence $O X Z$, only those components with the transmission coefficient $T_{\perp},\left(T_{\|}\right)$would be chosen in the incident fields [60]. $K$ denotes the component, parallel to the interface, of the wave vector $\mathbf{k}: k\left(\mathbf{s}_{x y}, s_{z}\right)=(K, 0, i q)$, $q=\sqrt{K^{2}-k^{2}}, k^{2}=K^{2}-q^{2}$.

\section{A. Reactive power and reactive helicity densities}

The densities of energy, $w=w_{e}+w_{m}$, and reactive power, $w_{\text {react }}=2 \omega\left(w_{m}-w_{e}\right),(\epsilon=\mu=1)$, of this wave are 


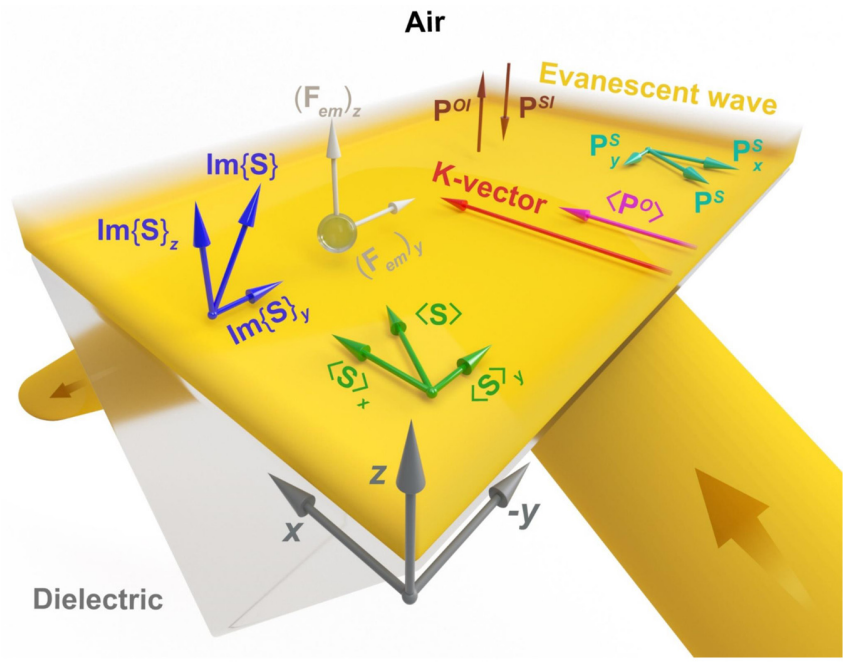

FIG. 1. The evanescent wave (yellow) of Eq. (19), with reactive power density given by (20), propagates in the air, parallel to the interface $z=0$ separating it from a dielectric in $z<0$, with $K$ vector along $O X$. Its amplitude decays as $\exp (-q z)$. The $\langle\mathbf{S}\rangle$ vector and spin momentum $\left\langle\mathbf{P}^{S}\right\rangle$, contained in $O X Y$, have $y$ components proportional to the wave helicity $\mathscr{H}$ (see Appendix C). The orbital momentum $\left\langle\mathbf{P}^{0}\right\rangle$ proportional to the energy density $w$ points along $O X$. The reactive momentum $\operatorname{Im}\{\mathbf{S}\}$, contained in $O Y Z$, and whose $z$ component is associated to the energy flux bouncing up and down along $O Z$ [cf. Eq. (28)], may be observed through the time-averaged force $\mathbf{F}_{\mathrm{em}}$, proportional to $\operatorname{Im}\{\mathbf{S}\}$, on a dipolar magnetodielectric particle placed on the interface. This force is due to the interference of the particle electric and magnetic induced dipoles. The lateral $y$ force, $\left(\mathbf{F}_{\mathrm{em}}\right)_{y}$, and transversal $z$ component, $\left(\mathbf{F}_{\mathrm{em}}\right)_{z}$, due to $\operatorname{Im}\{S\}_{y}$ and $\operatorname{Im}\{S\}_{z}$, are proportional to the wave reactive helicity, $\mathscr{H}_{\mathscr{R}}$, and reactive power density, $\boldsymbol{\nabla} \cdot \operatorname{Im}\{\mathbf{S}\}$, respectively. The imaginary orbital and spin momenta, $\mathbf{P}^{O I}$ and $\mathbf{P}^{S I}$, point along the $+z$ and $-z$ directions, respectively. The flow $\mathcal{F}_{\mathscr{H}_{\mathscr{R}}}$ of reactive helicity is, like $\operatorname{Im}\{\mathbf{S}\}$, in the $O Y Z$ plane with the $y$ component proportional to both the reactive power density, $w_{\text {react }}$, and the magnitude of the $K$ vector; while its $z$ component is proportional to the reactive helicity density $\mathscr{H}_{\mathscr{R}}$, so that across the $O X Y$ plane $\left(\mathcal{F}_{\mathscr{H}_{\mathscr{R}}}\right)_{z}$ holds the imaginary part of the complex helicity theorem, as shown by Eq. (30).

according to (2)

$$
\begin{aligned}
w & =\frac{1}{8 \pi} \frac{K^{2}}{k^{2}}\left(\left|T_{\perp}\right|^{2}+\left|T_{\|}\right|^{2}\right) \exp (-2 q z), \\
w_{\text {react }} & =\frac{c}{4 \pi} \frac{q^{2}}{k}\left(\left|T_{\perp}\right|^{2}-\left|T_{\|}\right|^{2}\right) \exp (-2 q z) .
\end{aligned}
$$

And the densities of helicity, $\mathscr{H}$, and reactive helicity, $\mathscr{H}_{\mathscr{R}}$, ( $\epsilon=\mu=1)$, of this evanescent wave are

$$
\begin{gathered}
\mathscr{H}=-\left(K^{2} / k^{3}\right) \operatorname{Im}\left\{T_{\perp} T_{\|}^{*}\right\} \exp (-2 q z) ; \\
\mathscr{H}_{\mathscr{R}}=\left(q^{2} / k^{3}\right) \operatorname{Re}\left\{T_{\perp} T_{\|}^{*}\right\} \exp (-2 q z) .
\end{gathered}
$$

\section{B. The reactive energy flow: Reactive momentum, imaginary spin, and imaginary orbital momenta}

The CPV is written as

$$
\mathbf{S}=\frac{c}{8 \pi \mu}\left[\frac{K}{k}\left(\left|T_{\perp}\right|^{2}+\left|T_{\|}\right|^{2}\right), \quad i 2 \frac{K q}{k^{2}} T_{\perp}^{*} T_{\|},\right.
$$

$$
\begin{aligned}
& \left.-i \frac{q}{k}\left(\left|T_{\perp}\right|^{2}-\left|T_{\|}\right|^{2}\right)\right] \exp (-2 q z) \\
= & {\left[\frac{c k}{K} w, \frac{c}{4 \pi}\left(-\frac{k q}{K} \mathscr{H}+i \frac{k K}{q} \mathscr{H}_{\mathscr{R}}\right),-i \frac{1}{2 \mu q} w_{\text {react }}\right] . }
\end{aligned}
$$

For the sake of comprehensiveness, in Appendix C, we present the well-known main time-averaged quantities. Here we concentrate on those reactive less known and their interrelations.

The reactive, or imaginary, part of the CPV is

$$
\begin{aligned}
\operatorname{Im}\{\mathbf{S}\}= & \frac{c}{8 \pi \mu}\left[0,2 \frac{K q}{k^{2}} \operatorname{Re}\left\{T_{\perp} T_{\|}^{*}\right\}, \frac{q}{k}\left(\left|T_{\|}\right|^{2}-\left|T_{\perp}\right|^{2}\right)\right] \\
& \times \exp (-2 q z)=\frac{1}{2 q}\left[0, \frac{c k K}{2 \pi} \mathscr{H}_{\mathscr{R}},-w_{\text {react }}\right],
\end{aligned}
$$

which yields the reactive or imaginary momentum of the field (sometimes called imaginary Poynting momentum [22,25]), $\mathbf{g}^{I}=\operatorname{Im}\{\mathbf{g}\}=\operatorname{Im}\{\mathbf{S}\} / c^{2}$ :

$$
\begin{aligned}
\mathbf{g}^{I}= & \frac{1}{8 \pi c}\left[0,2 \frac{K q}{k^{2}} \operatorname{Re}\left\{T_{\perp} T_{\|}^{*}\right\}, \frac{q}{k}\left(\left|T_{\|}\right|^{2}-\left|T_{\perp}\right|^{2}\right)\right] \\
& \times \exp (-2 q z)=\frac{1}{2 q c^{2}}\left[0, \frac{c k K}{2 \pi} \mathscr{H}_{\mathscr{R}},-w_{\text {react }}\right],
\end{aligned}
$$

whose components come from two vectors that we put forward next: the density of both reactive spin momentum $\mathcal{P}^{S}$ and reactive orbital momentum $\mathcal{P}^{O}$ :

$$
\begin{aligned}
& \mathcal{P}^{S}=\frac{1}{2}\left(\mathbf{P}_{m}^{S I}-\mathbf{P}_{e}^{S I}\right)=\frac{K}{2 c q}\left(0, \frac{k}{2 \pi} \mathscr{H}_{\mathscr{R}},-\frac{K}{c k^{2}} w_{\text {react }}\right), \\
& \mathcal{P}^{O}=\frac{1}{2}\left(\mathbf{P}_{m}^{O I}-\mathbf{P}_{e}^{O I}\right)=\frac{q}{2 c q}\left(0,0, \frac{q}{c k^{2}} w_{\text {react }}\right) .
\end{aligned}
$$

Namely,

$$
\mathbf{g}^{I}=\mathcal{P}^{S}+\mathcal{P}^{O}
$$

The electric and magnetic imaginary spin and orbital momenta of (25) are given in Appendix C, Eqs. (C-10)- (C-14). We emphasize that, as seen in (24)-(26), $g_{y}^{I}$ is fully due to $\mathcal{P}_{y}^{S}$, while $g_{z}^{I}$ comes from $\mathcal{P}_{z}^{S}+\mathcal{P}_{z}^{O}$. Note that, interestingly and in contrast with Eq. (C6), denoting $\mathbf{P}^{S I}=(1 / 2)\left(\mathbf{P}_{e}^{S I}+\mathbf{P}_{m}^{S I}\right)=$ $\left(0,0,-\frac{q}{k c} w\right), \quad \mathbf{P}^{O I}=(1 / 2)\left(\mathbf{P}_{e}^{O I}+\mathbf{P}_{m}^{O I}\right)=\left(0,0, \frac{q}{k c} w\right)$, one

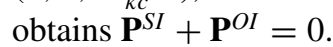

\section{The reactive helicity flow}

In turn, the reactive helicity flow [cf. Eq. (15)] is

$$
\begin{aligned}
\mathcal{F}_{\mathscr{H}_{\mathscr{R}}}= & \frac{c}{4 k}\left[0, \frac{2 K q}{k^{2}}\left(\left|T_{\|}\right|^{2}-\left|T_{\perp}\right|^{2}\right),\right. \\
& \left.-\frac{4 q}{k} \operatorname{Re}\left\{T_{\perp} T_{\|}^{*}\right\}\right] \exp (-2 q z) \\
= & -\frac{1}{q}\left[0, \frac{2 \pi K}{k^{2}} w_{\text {react }}, c k \mathscr{H}_{\mathscr{R}}\right] .
\end{aligned}
$$

It should be emphasized that although both $\operatorname{Im}\{\mathbf{S}\}$ and $\mathcal{F}_{\mathscr{H}_{\mathscr{R}}}$ have a $y$ component proportional to $K>k$ (as well as proportional to $\mathscr{H}_{\mathscr{R}}$ in the former and to $w_{\text {react }}$ in the latter), there is no case of superluminal propagation for these quantities since both are alternating flows with zero time average. We show 
below that $\mathcal{F}_{z}$ represents up and down flow of reactive helicity in the $O Z$ direction, matching with the imaginary part of the complex helicity theorem, Eq. (16).

From the above equations, it is important to remark that while the time-averaged energy and helicity densities are linked to $\langle\mathbf{S}\rangle,\langle\mathbf{g}\rangle,\langle\mathcal{S}\rangle,\left\langle\mathbf{P}^{S}\right\rangle$, and $\left\langle\mathbf{P}^{O}\right\rangle$, the densities of reactive power, $w_{\text {react }}$, and reactive helicity, $\mathscr{H}_{\mathscr{R}}$, are exclusive of $\operatorname{Im}\{\mathbf{S}\}, \mathbf{g}^{I}$, and $\mathcal{F}_{\mathscr{H}_{\mathscr{R}}}$.

We show below that the $z$ component of $\operatorname{Im}\{\mathbf{S}\}$, which matches with the imaginary part of the complex Poynting theorem, is associated with an up and down flow of reactive power in the decay $z$ direction of the evanescent wave, and hence it is not a net flow of energy. Analogously, the same happens with the $z$ component of $\mathcal{F}_{\mathscr{H}_{\mathscr{R}}}$. However, as seen below, both the $y$ and $z$ components of $\operatorname{Im}\{\mathbf{S}\}$ produce detectable optical forces and, hence, make the reactive quantities $w_{\text {react }}$ and $\mathscr{H}_{\mathscr{R}}$ observable.

\section{Reactive power conservation law}

The $z$ component of $\operatorname{Im}\{\mathbf{S}\}$ [cf. Eq. (23)] depends on the reactive power density, $\boldsymbol{\nabla} \cdot \operatorname{Im}\{\mathbf{S}\}$, of the evanescent wave in the half-space $z>0$ (which actually concentrates in the near field region above the interface $z=0$, namely, at $z \ll \lambda$ ) flowing back and forth along $O Z$ at twice the frequency $\omega$, without contributing to a net energy flow since its time average is zero. That is, one has from the CPV theorem:

$$
\begin{aligned}
\nabla \cdot \operatorname{Im}\{\mathbf{S}\} & \equiv \partial_{z} \operatorname{Im}\{\mathbf{S}\}=w_{\text {react }} \\
& \equiv-2 q \operatorname{Im}\{\mathbf{S}\}_{z},
\end{aligned}
$$

which obviously agrees with (23). Therefore taking (28) into account, the total reactance [12] of the dielectric-air interface system associated to the evanescent wave is

$$
\int_{V} d^{3} r \nabla \cdot \operatorname{Im}\{\mathbf{S}\}=\Sigma \int_{0}^{\infty} d z \nabla \cdot \operatorname{Im}\{\mathbf{S}\}=-\operatorname{Im}\left\{\mathbf{S}_{z}(z=0)\right\} .
$$

$\Sigma$ being the area of the $X Y$ plane resulting from the volume integration.

\section{E. Reactive helicity conservation law}

Analogously, the $z$ component of $\mathcal{F}_{\mathscr{H}_{\mathscr{R}}}$, Eq. (27), depends on the reactive helicity density, $\nabla \cdot \mathcal{F}_{\mathscr{H}_{\mathscr{R}}}$, of the evanescent wave in the half-space $z>0$, concentrated in the near field on $z=0$, flowing up and down in the the $z$ direction without yielding a net flow since its time average is zero. That is, in agreement with Eq. (14) $(\mathbf{j}=0)$,

$$
\begin{aligned}
\nabla \cdot \mathcal{F}_{\mathscr{H}_{\mathscr{R}}} & \equiv \partial_{z} \mathcal{F}=\frac{2 c q^{2}}{k^{2}} \operatorname{Re}\left\{T_{\perp} T_{\|}^{*}\right\} \\
& =-2 q\left(\mathcal{F}_{\mathscr{H}_{\mathscr{R}}}\right)_{z} \equiv 2 \omega \mathscr{H}_{\mathscr{R}} .
\end{aligned}
$$

Hence, the $z$ component of $\mathcal{F}_{\mathscr{H}_{\mathscr{R}}}$ is proportional to the reactive power density.

It is evident that while reactive power and reactive helicity exist in evanescent waves [see also Eqs. (7) of Sec. II and (18) of Sec. III] and in standing waves [3,24], they do not exist in plane propagating waves, whatever their polarization be. Therefore reactive helicity, like reactive en- ergy, exists in the near field region of scattering or emitting objects.

\section{F. Observability of the transversal and perpendicular} components of the imaginary momentum $\mathrm{g}^{I}$ : Optical forces on a magnetodielectric dipolar particle due to the reactive helicity and reactive power

Let a magnetodielectric dipolar particle be placed on the $z=0$ interface. An illuminating wavefield, and in particular the evanescent wave, exerts an optical force on it due to the interaction between its induced electric (e) and magnetic (m) dipoles [21], viz.,

$$
\left\langle\mathbf{F}_{\mathrm{e}-\mathrm{m}}\right\rangle=\frac{8 \pi k^{4} c}{3}\left[-\operatorname{Re}\left(\alpha_{e} \alpha_{m}^{*}\right)\langle\mathbf{g}\rangle+\operatorname{Im}\left(\alpha_{e} \alpha_{m}^{*}\right) \mathbf{g}^{I}\right] .
$$

The e and m polarizabilities $\alpha_{e}$ and $\alpha_{m}$ are related with the $a_{1}$ and $b_{1}$ Mie coefficients of the field scattered by the particle by [21] $\alpha_{e}=i 3 a_{1} / 2 k^{3}$ and $\alpha_{m}=i 3 b_{1} / 2 k^{3}$.

The first term of (31), proportional to $\langle\mathbf{g}\rangle$, has been studied (see its main features in Appendix C). Here we are interested in the second term that contains the reactive momentum $\mathbf{g}^{I}$.

The $y$-component $\left\langle\mathbf{F}_{\mathrm{e}-\mathrm{m}}^{\mathbf{g}^{I}}\right\rangle_{y}$ due to $g_{y}^{I}$ of (31) was obtained in Ref. [22], being considered by the authors "a quite intriguing result" characterized through the second Stokes parameter with a rather small contribution in measurements of $\left\langle\mathbf{F}_{\mathrm{e}-\mathrm{m}}\right\rangle_{y}$. We have shown above that this force has a reactive origin since it is fully due to the reactive spin $y$ component, being characterized by the reactive helicity $\mathscr{H}_{\mathscr{R}}$. Furthermore, we establish here that $\left\langle\mathbf{F}_{\mathrm{e}-\mathrm{m}}^{\mathbf{g}^{I}}\right\rangle_{y}$ is detectable since it may widely exceed the known component $\left\langle\mathbf{F}_{\mathrm{e}-\mathrm{m}}^{\langle\mathbf{g}\rangle}\right\rangle_{y}$ due to $\langle g\rangle_{y}$.

For instance, Fig. 2(a) shows forces on a $\mathrm{Si}$ spherical particle placed on the interface $z=0:\left\langle\mathbf{F}_{\mathrm{e}-\mathrm{m}}^{\mathbf{g}^{I}}\right\rangle_{y}$ compared with $\left\langle\mathbf{F}_{\mathrm{e}-\mathrm{m}}^{\langle\mathbf{g}\rangle}\right\rangle_{y}$. The particle electric and magnetic dipole resonances are at $\lambda_{e}=492 \mathrm{~nm}$ and $\lambda_{m}=668 \mathrm{~nm}$, respectively [see Fig. 4(a)], and the second Kerker condition (K2) wavelength (at which the particle scatters minimum forward intensity, see Sec. V B) is $\lambda_{K 2}=608 \mathrm{~nm}$. As seen, the magnitude of $\left\langle\mathbf{F}_{\mathrm{e}-\mathrm{m}}^{\mathbf{g}^{I}}\right\rangle_{y}$ is much greater than $\left\langle\mathbf{F}_{\mathrm{e}-\mathrm{m}}^{\langle\mathbf{g}\rangle}\right\rangle_{y}$ near $\lambda_{e}$ and $\lambda_{m}$ where the latter changes sign (see the sharp asymptotic values of the ratio between both forces). Thus $\left\langle\mathbf{F}_{\mathrm{e}-\mathrm{m}}^{\mathbf{g}^{I}}\right\rangle_{y}$, which remains negative, should be detectable near these resonances. Besides, in the proximities of K2, i.e., of $\lambda_{K 2},\left\langle\mathbf{F}_{\mathrm{e}-\mathrm{m}}^{\mathbf{g}^{I}}\right\rangle_{y}=-\left\langle\mathbf{F}_{\mathrm{e}-\mathrm{m}}^{\langle\mathbf{g}\rangle}\right\rangle_{y}$.

While $\left\langle\mathbf{F}_{\mathrm{e}-\mathrm{m}}^{\langle\mathbf{g}\rangle}\right\rangle_{z}=0$, one observes in Fig. 2(b) features of the perpendicular force $\left\langle\mathbf{F}_{\mathrm{e}-\mathrm{m}}^{\mathbf{g}^{I}}\right\rangle_{z}$ similar to those of its $y$ component comparing it with the gradient force [61,62], which by choosing $T_{\|}=0$ reads $\left\langle\mathbf{F}^{\mathrm{grad}}\right\rangle_{z}=-(1 / 2) q\left[\alpha_{e}^{R}\left|T_{\perp}\right|^{2}+\right.$ $\left.\alpha_{m}^{R}\left|T_{\perp}\right|^{2}\left(2 K^{2} / k^{2}-1\right)\right](0,0,1)$. The superscript $R$ stands for real part. Again, the sharp ratio between both near $\lambda=665$ $\mathrm{nm}$ indicates that the wavelength zone where $\left\langle\mathbf{F}_{\mathrm{e}-\mathrm{m}}^{\mathbf{g}^{I}}\right\rangle_{z}$, which remains positive, may be detected. Furthermore, the bump of this ratio in the proximities of $\lambda=520 \mathrm{~nm}$ shows that this force is over twice the gradient force.

We conclude thereby that there exists a measurable transverse $y$ component of $\left\langle\mathbf{F}_{\mathrm{e}-\mathrm{m}}\right\rangle$ due to the reactive spin momentum density and hence to $\mathscr{H}_{\mathscr{R}}$, which may be dom- 
(a)

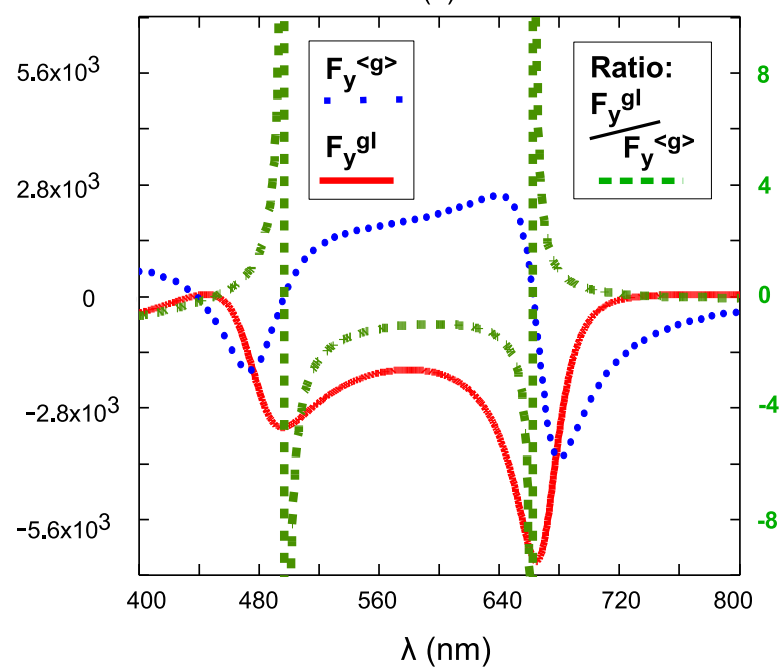

(b)

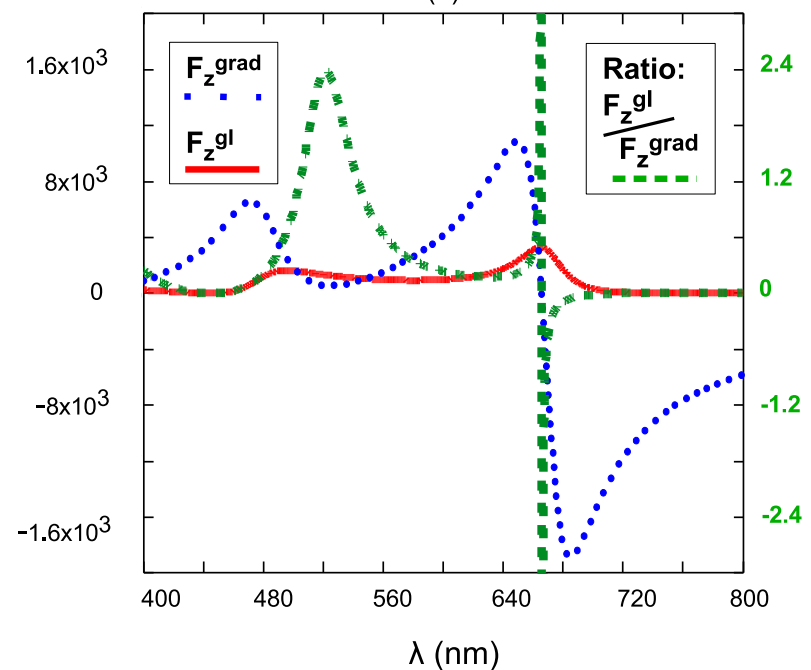

FIG. 2. An evanescent wave (see Fig. 1) is created in $z \geqslant 0$ by TIR at the interface $z=0$ of a linearly polarized plane propagating wave of complex amplitudes $A_{\perp}$ and $A_{\|}$in a dielectric of refractive index $n=1.5$ with angle of incidence $60^{\circ}$. A Si spherical particle of radius $a=75 \mathrm{~nm}$ is deposited on the interface. Its electric and magnetic dipole resonances are at $\lambda_{e}=492 \mathrm{~nm}$ and $\lambda_{m}=668 \mathrm{~nm}$, respectively [cf. Fig. 4(a)]. This wave exerts on the particle the following transversal and perpendicular optical forces: (a) with $A_{\perp}=A_{\|}$, force transversal components, $\left\langle\mathbf{F}_{\mathrm{e}-\mathrm{m}}^{\mathbf{g}^{I}}\right\rangle_{y}$ due to $g_{y}^{I},\left\langle\mathbf{F}_{\mathrm{e}-\mathrm{m}}^{\langle\mathbf{g}\rangle}\right\rangle_{y}$ from $\left\langle g_{y}\right\rangle$, and ratio $\left\langle\mathbf{F}_{\mathrm{e}-\mathrm{m}}^{\mathbf{g}^{I}}\right\rangle_{y} /\left\langle\mathbf{F}_{\mathrm{e}-\mathrm{m}}^{\langle\mathbf{g}\rangle}\right\rangle_{y}$ whose values correspond to the right ordinate axis. (b) Choosing $A_{\|}=0, A_{\perp} \neq 0$ : normal component $\left\langle\mathbf{F}_{\mathrm{e}-\mathrm{m}}^{\mathbf{g}^{I}}\right\rangle_{z}$ due to $g_{z}^{I}$, gradient force $\left\langle\mathbf{F}^{\mathrm{grad}}\right\rangle_{z}$, and ratio $\left\langle\mathbf{F}_{\mathrm{e}-\mathrm{m}}^{\mathbf{g}^{I}}\right\rangle_{z} /\left\langle\mathbf{F}^{\mathrm{grad}}\right\rangle_{z}$ whose values correspond to the right ordinate axis. Notice that the positive values of the gradient force, repelling the particle from the interface, are due to negative values of $\alpha_{e}^{R}$ and/or $\alpha_{m}^{R}$. The bump of $\left\langle\mathbf{F}^{\text {grad }}\right\rangle_{z}$ near $\lambda_{e}$ and its steep change of sign close to $\lambda_{m}$, are due to the gradient force felt by the induced electric and magnetic dipoles, respectively. The wavelength at which the second Kerker condition holds is $\lambda_{K 2}=608 \mathrm{~nm}$. The force (normalized to area) units are $\mathrm{fN} / \mu \mathrm{m}^{2}$ for an incident power of $1 \mathrm{~mW} / \mathrm{mm}^{2}$.

inant upon the transversal component of $\left\langle\mathbf{F}_{\mathrm{e}-\mathrm{m}}\right\rangle$ stemming from the field (Poynting) momentum, namely from $\mathscr{H}$. Hence $\mathscr{H}_{\mathscr{R}}$ is observable. Besides, the normal force $\left\langle\mathbf{F}_{\mathrm{e}-\mathrm{m}}\right\rangle_{z}$, which is exclusively due $g_{z}^{I}$, ( since $\left\langle g_{z}\right\rangle=0$ ), characterized by the reactive power density $w_{\text {react }}$ of the evanescent wave, has not yet been addressed as far as we know, and may be detected at wavelengths at which, as seen above, clearly exceeds the gradient force, making $w_{\text {react }}$ also an observable quantity.

\section{CASE 2: REACTIVE POWER AND REACTIVE HELICITY FROM A MAGNETODIELECTRIC DIPOLAR SPHERE}

We consider a magnetodielectric spherical particle of radius $a$ and volume $V_{0}$, dipolar in the wide sense (see Sec. IVF), namely whose electric and magnetic polarizabilities are given by the first electric and magnetic Mie coefficients, respectively [21,36], in air. We first address the reactive power and stored energy of this magnetoelectric dipole with electric and magnetic moments $\mathbf{p}$ and $\mathbf{m}$, respectively. For a wave, $\mathbf{E}^{(i)}, \mathbf{B}^{(i)}$, incident on the particle centered at $\mathbf{r}=\mathbf{0}$, the dipolar moments are $\mathbf{p}=\alpha_{e} \mathbf{E}^{(i)}(\mathbf{0})$ and $\mathbf{m}=$ $\alpha_{m} \mathbf{B}^{(i)}(\mathbf{0})$.

\section{A. Reactive power and stored energy}

Concerning the CPV, $\mathbf{S}^{(\mathbf{s})}=\frac{c}{8 \pi} \mathbf{E}^{(s)} \times \mathbf{H}^{(s) *}$, of the emitted fields, (cf. Appendix D), we are interested in its radial component, $\mathbf{S}^{(s)} \cdot \hat{\mathbf{r}}$, across a spherical surface $\partial V$ of radius $r$ concentric with the particle and enclosing it. Using the fields of Appendix E, this is straightforwardly integrated, yielding

$$
\int_{\partial V} \mathbf{S}^{(s)} \cdot \hat{\mathbf{r}} d^{2} r=\frac{c k^{4}}{3}\left(|\mathbf{p}|^{2}+|\mathbf{m}|^{2}\right)+\frac{i c k}{3 r^{3}}\left(|\mathbf{p}|^{2}-|\mathbf{m}|^{2}\right),
$$

whose real part, $\int_{\partial V}\left\langle\mathbf{S}^{(\mathbf{s})}\right\rangle \cdot \hat{\mathbf{r}} d^{2} r=\frac{c k^{4}}{3}\left(|\mathbf{p}|^{2}+|\mathbf{m}|^{2}\right)$ is the well-known radiated (scattered) total power $W^{(s)}$ independent of the distance $r$ to the center $r=0$ and corresponds to the wavefield reradiated up to the far zone, i.e., with the $r^{-1}$ dependence [cf. Appendix D, Eqs. (D-1) and (D-2)].

Here we are particularly interested in the imaginary part

$$
\left.\int_{\partial V} \operatorname{Im}\left\{\mathbf{S}^{(s)}\right\} \cdot \hat{\mathbf{r}} d^{2} r=\frac{c k}{3 r^{3}}\left(|\mathbf{p}|^{2}-|\mathbf{m}|^{2}\right)\right]=-W_{\text {react }}^{(s)},
$$

where $W_{\text {react }}^{(s)}$ is the reactive power outside $V$. It arises from the near and intermediate fields: $\mathbf{E}^{(s)}(\mathbf{r})=\left\{\frac{1}{\epsilon r^{3}}[3 \mathbf{n}(\mathbf{n}\right.$. $\left.\mathbf{p})-\mathbf{p}]-\sqrt{\frac{\mu}{\epsilon}}(\mathbf{n} \times \mathbf{m}) \frac{i k}{r^{2}}\right\} e^{i k r}$, and $\mathbf{B}^{(s)}(\mathbf{r})=\left\{\frac{\mu}{r^{3}}[3 \mathbf{n}(\mathbf{n} \cdot \mathbf{m})-\right.$ $\left.\mathbf{m}]+\sqrt{\frac{\mu}{\epsilon}}(\mathbf{n} \times \mathbf{p}) \frac{i k}{r^{2}}\right\} e^{i k r}$. (We recall that the expression $\frac{i c k}{3 r^{3}}|\mathbf{p}|^{2}$ for a purely electric dipolar emitter is well-known in antenna theory $[3,5,12]$.) We note that the $r^{-3}$ dependence of $W_{\text {react }}^{(s)}$ makes it to acquire much larger values than $W^{(s)}$ in the near field region.

The reactive power $W_{\text {react }}^{(s)}$ is the difference between averaged stored magnetic and electric powers, which dominate in the near and intermediate field regions around the particle, and that do not propagate. To see it, we write the mean stored electric and magnetic energy densities as $[8,10,11]\left\langle\tilde{w}_{e}^{(s)}\right\rangle=$ 
$\left\langle w_{e}^{(s)}\right\rangle-\left\langle w_{e}^{(\mathrm{FF})}\right\rangle,\left\langle\tilde{w}_{m}^{(s)}\right\rangle=\left\langle w_{m}^{(s)}\right\rangle-\left\langle w_{m}^{(\mathrm{FF})}\right\rangle$. While $\left\langle w_{e}^{(s)}\right\rangle$ and $\left\langle w_{m}^{(s)}\right\rangle$ are the energy densities of the full fields $\mathbf{E}^{(s)}$ and $\mathbf{B}^{(s)}$, i.e., with all terms of their expressions (D-1)-(D-4) of Appendix D; the supescript (FF) stands for far zone electric and magnetic energy densities, i.e, corresponding to the fields (D-1)-(D-4) with only terms of $r^{-1}$ dependence. Since evidently $\left\langle w_{m}^{(\mathrm{FF})}\right\rangle=\left\langle w_{e}^{(\mathrm{FF})}\right\rangle$, we have that $\left\langle w_{m}^{(s)}\right\rangle-\left\langle w_{e}^{(s)}\right\rangle=$ $\left\langle\tilde{w}_{m}^{(s)}\right\rangle-\left\langle\tilde{w}_{e}^{(s)}\right\rangle[63]$.

Then we write $W_{\text {react }}^{(s)}$ in terms of the volume integral outside the volume $V$ in the above mentioned sphere $\partial V$ of radius $r$, centered in $\mathbf{r}=\mathbf{0}$ :

$$
\begin{aligned}
W_{\text {react }}^{(s)} & \left.=\frac{c k}{3 r^{3}}\left(|\mathbf{m}|^{2}-|\mathbf{p}|^{2}\right)\right] \\
& =2 \omega \int_{V_{\infty}-V} d^{3} r\left(\left\langle w_{m}^{(s)}\right\rangle-\left\langle w_{e}^{(s)}\right\rangle\right) \\
& =2 \omega \int_{V_{\infty}-V} d^{3} r\left(\left\langle\tilde{w}_{m}^{(s)}\right\rangle-\left\langle\tilde{w}_{e}^{(s)}\right\rangle\right),
\end{aligned}
$$

where $V_{\infty}$ is the volume of a large sphere $(k r \rightarrow \infty)$. Making $V=V_{0}$ and $r=a$, (34) yields the reactive power outside the particle. The proof of (34) is given in Appendix E [cf. Eq. (E3)]. We anticipate that Eq. (34) is a consequence of the optical theorem for reactive power, which we put forward in Sec. VI [cf. Eq. (47)].

As shown in Eq. (3), $\int_{\partial V} \operatorname{Im}\left\{\mathbf{S}^{(s)}\right\} \cdot \hat{\mathbf{r}} d^{2} r$ is associated to an instantaneous energy flow alternating back and forth from the scatterer without losses, at frequency $2 \omega$, with zero net energy transport in the embedding vacuum. Nonetheless, this alternating flow builds $W_{\text {react }}^{(s)}$ according to Eq. (33), also deduced from Eq. (34). As a consequence, there is an accretion of timeaveraged nonpropagating reactive power and stored energy, $W^{\text {(sto) }}$, outside $V$. (We note a concept analogous to $W^{\text {(sto) }}$ for purely electric dipolar RF antennas, e.g., [3,8,10,11].) Taking $V=V_{0}$, the total energy stored outside the particle is obtained by

$$
\begin{aligned}
W^{(\text {sto })}= & \int_{V_{\infty}-V_{0}}\left(\left\langle\tilde{w}_{m}^{(s)}\right\rangle+\left\langle\tilde{w}_{e}^{(s)}\right\rangle\right) d^{3} r \\
= & \int_{V_{\infty}-V_{0}}\left(\left\langle w_{m}^{(s)}\right\rangle+\left\langle w_{e}^{(s)}\right\rangle\right) d^{3} r \\
& -\int_{V_{\infty}-V_{0}}\left(\left\langle\tilde{w}_{m}^{(\mathrm{FF})}\right\rangle+\left\langle\tilde{w}_{e}^{(\mathrm{FF})}\right\rangle\right) d^{3} r \\
= & \frac{1}{6}\left(|\mathbf{p}|^{2}+|\mathbf{m}|^{2}\right)\left(\frac{1}{a^{3}}+\frac{2 k^{2}}{a}\right) .
\end{aligned}
$$

Appendix E provides the proof of (35) [cf. Eq. (E4)].

The quality factor associated with $W^{\text {(sto) }}$ is $Q=\frac{2 \omega W^{(\text {sto })}}{W^{(s)}}=$ $\frac{1}{(k a)^{3}}+\frac{2}{k a}$. Hence being independent of the strength of the electric and/or magnetic dipole moments.

Since in general a dipolar particle in the wide sense cannot be abstracted as a point dipole, the overall interior reactive power, $W_{\text {react, int }}^{(s)}$, and the interior stored energy, $W^{(\text {sto,int })}$, obtained in a similar way to (34) and (35), but by integrating in $V_{0}$ the mean energies of the interior field, are also of interest. For a linearly, or circularly, polarized incident plane wave of unit intensity, a straightforward calculation yields $\left(k c / 3 a^{3}\right)\left(9 / 4 k^{6}\right)\left(0.055\left|d_{1}\right|^{2} \mp 0.018\left|c_{1}\right|^{2}\right)$. The upper and lower sign in $\mp$ apply to $W_{\text {react }}^{(s)}$ int and $W^{(\text {sto,int) }}$, respectively, while $d_{1}$ and $c_{1}$ are the first electric and magnetic Mie coefficients of the fields inside the particle (e.g., Eq. (4.45) of Ref. [64]). Notice the appearance of $\left|d_{1}\right|^{2}$ and $\left|c_{1}\right|^{2}$ in $W_{\text {react, int }}^{(s)}$ with sign opposite to that of $|\mathbf{p}|^{2}$ and $|\mathbf{m}|^{2}$ in (34), i.e., of the first electric and magnetic external Mie coefficient squared moduli, $\left|a_{1}\right|^{2}$ and $\left|b_{1}\right|^{2}$, in $W_{\text {react }}^{(s)}$; we shall see that this has consequences for the total (i.e., interior plus external) reactive power at resonant wavelengths.

\section{B. Reactive power, resonances, and Kerker conditions}

Figure 3 depicts $W_{\text {react }}^{(s)}(\lambda, a), \quad W_{\text {react, int }}^{(s)}(\lambda, a), \quad$ and $W_{\text {react }, T}^{(s)}(\lambda, a)=W_{\text {react }}^{(s)}(\lambda, a)+W_{\text {react, int }}^{(s)}(\lambda, a)$ for $\mathrm{Si}$ spheres within the range of wavelengths where they become dipolar magnetodielectric, and for different size radii $a$, taking advantage of their scaling property with their impact parameter (see Fig. 2 of Ref. [36] and Ref. [46]). The redshift of the electric and magnetic dipole resonances $\lambda_{e}$ and $\lambda_{m}$ as $a$ grows, observed in the scattering cross-section [40,46], [see also $W^{(s)}$ in Fig. 3(b)], is observed in the peaks or dips of these reactive powers as they are much influenced by these resonances. An important feature of these surfaces is that the total reactive power vanishes, changing its sign, close to the resonance wavelengths where $W^{(s)}$ is maximum, irrespective of $a$. This is detailed in Fig. 4(b) on a cross sectional plane $a=75 \mathrm{~nm}$ of these surfaces, depicting the scattered power, along with the external, interior, and total reactive powers. Also Fig. 4(c) shows the stored energies, while in Fig. 4(a), one sees the electric and magnetic external first Mie coefficients $a_{1}$ and $b_{1}$ with resonant maxima at $\lambda_{e}=492 \mathrm{~nm}$ and $\lambda_{m}=668 \mathrm{~nm}$, respectively, and the internal coefficients, $d_{1}$ and $c_{1}$.

The vanishing of the total reactive power, $W_{\text {react }, T}^{(s)}$, observed in Figs. 3(c) and 4(b) close to the electric and magnetic resonances $\lambda_{e}$ and $\lambda_{m}$ where the scattered power, $W^{(s)}$, is produced with maximum efficiency, is quite relevant because it manifests a cancellation between the internal and external reactive power peaks; the stored energies [cf. Fig. 4(c)] being also resonant near these wavelengths. In fact, this matches [31] with knowledge from RF antenna theory according to which a maximum radiation efficiency is sought by minimizing their reactive power and $Q$ factor [3,6-8,10-12], even though in these works the capacitive-electric dipole wavelength $\lambda_{e}$ : $\mathbf{E}$ dominates in $r>a, r<\lambda_{e}$ (inductive: magnetic dipole wavelength $\lambda_{m}$ : $\mathbf{B}$ dominates in $r>a, r<\lambda_{m}$ )—nature of $W_{\text {react }}^{(s)}$ is compensated and tuned to resonance by adding an inductive (capacitive) storage element.

Such element, here in the optics domain, is provided by the particle interior through the emergence of a dominant $\mathbf{B}$ at $\lambda_{e}$ (dominant $\mathbf{E}$ at $\left.\lambda_{m}\right)$ of $W_{\text {react, int }}^{(s)}$ inside the particle, $(r<$ a). By the same token the total stored energies have peaks in these resonant wavelengths $\lambda_{e}$ and $\lambda_{m}$, like the scattered power $W^{(s)}$ [cf. Fig. 4(c)], being evident that the difference of external and internal stored energies: $W^{\text {(sto,Dif) }}=W^{\text {(sto) }}$ $W^{\text {(sto,int) }}$ vanishes, like $W_{\text {react, } T}^{(s)}$, close to $\lambda_{e}$ and $\lambda_{m}$.

These results illustrate the concepts of reactive and stored power in and around a magnetodielectric, or Huygens, particle when choosing configurations and wavelengths such that the 
(a)

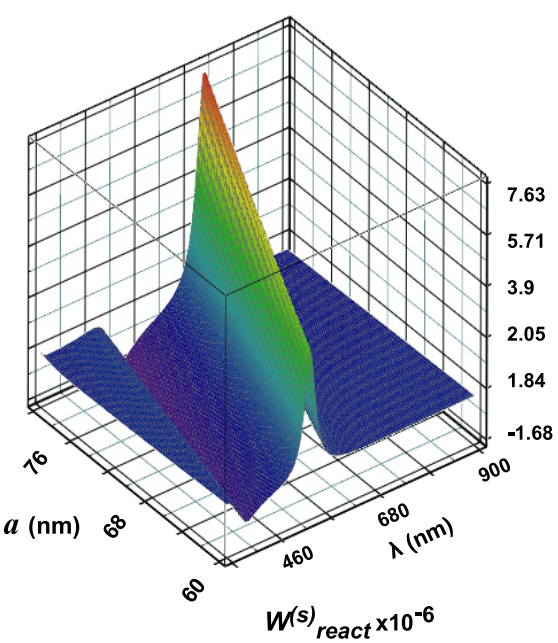

(b)

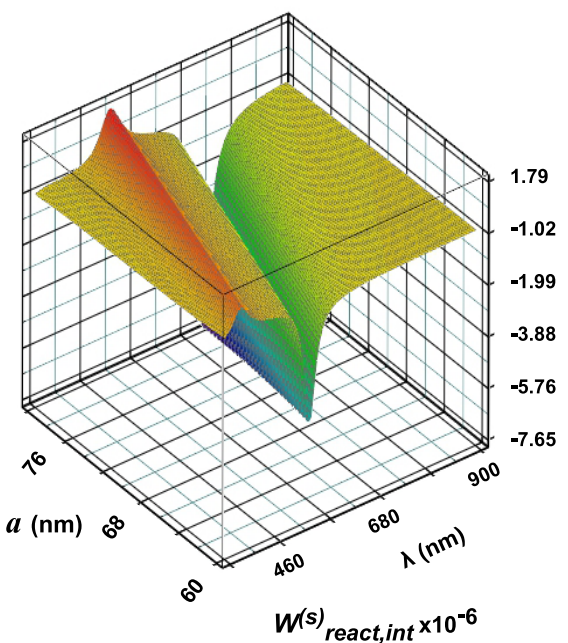

(c)

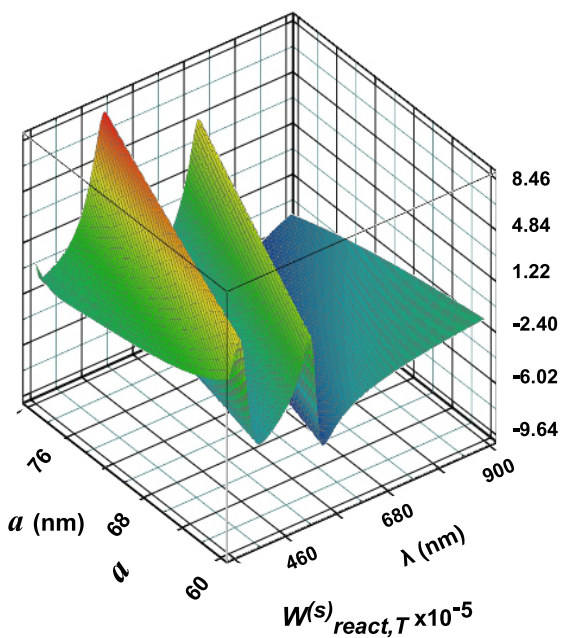

FIG. 3. 3D graphs of reactive powers as functions of wavelength $\lambda$ and radius $a$ of a magnetodielelectric sphere of Si in air, illuminated by a (linearly or circularly polarized) plane wave. (a): External $W_{\text {react }}^{(s)}$. (b): Interior $W_{\text {react,int }}^{(s)}$. (c): Total $W_{\text {react, } T}^{(s)}=W_{\text {react }}^{(s)}+W_{\text {react,int }}^{(s)}$. The sign of $W_{\text {react,int }}^{(s)}$ is opposite to that of $W_{\text {react }}^{(s)}$ for all $\lambda$ and $a$. The dip and peak of $W_{\text {react }}^{(s)}$ correspond to the electric and magnetic resonance, respectively, and are close to these resonances in $W_{\text {react,int }}^{(s)}$. Like the resonances exhibited by the scattered power $W^{(s)}$ [46], these peaks and dips are redshifted as $a$ increases. $W_{\text {react, } T}^{(s)}$ vanishes in the proximities of these resonances, irrespective of $a$.

accretion of external reactive power and stored energy in the particle near field, through the IPV alternating flow, be as large as possible; thus scattering with maximum efficiency $W^{(s)}$ and possessing the highest possible $Q$ factor for applications in light-matter interactions, while its total reactive power $W_{\text {react, } T}^{(s)}$ vanishes or is near zero at resonant wavelengths. Since, however, these magnetodielectric nanoresonators have rather low $Q$ 's $(Q \leqslant 7)$, external (and internal) stored power enhancements may be achieved either by sets of such magnetodielectric particles, even metal coated, using them as building blocks of photonic molecules, metasurfaces [65], or in regimes of bound states in the continuum $[35,66]$.

Returning to Fig. 4(a), we observe the lines $\left|a_{1}\right|^{2}$ and $\left|b_{1}\right|^{2}$ crossing each other at the two Kerker wavelengths: $\lambda_{K 1}=$
$738.5 \mathrm{~nm}$ and $\lambda_{K 2}=608 \mathrm{~nm}$, which correspond to the first Kerker condition (K1), (zero backscattering, $\alpha_{m}=\alpha_{e},|\mathbf{p}|=$ $|\mathbf{m}|$ ), and second Kerker condition (K2), (minimum forward scattering, $\left.\alpha_{m}=-\alpha_{e}^{*},|\mathbf{p}| \simeq|\mathbf{m}|\right)[37,39,40,44-47]$. One sees in Fig. 4(b) that at $\lambda_{K 1}, W_{\text {react }}^{(s)}=0$ in accordance with Eqs. (33) and (34), $W_{\text {react,int }}^{(s)} \simeq 0$; while both $W_{\text {react }}^{(s)}$ and $W_{\text {react,int }}^{(s)} \simeq 0$ at $\lambda_{K 2}$. Also notice in Fig. 4(c) that the stored energies, $W^{\text {(sto })}$ and $W^{\text {(sto,int) }}$, and scattered power, $W^{(s)}$, are near minimum in the proximities of $\lambda_{K 1}$ and $\lambda_{K 2}$. These features happen in Fig. 3 for any $a$. Therefore the analysis based on the particle reactive power allows to envisage the Kerker conditions, K1 and $\mathrm{K} 2$, from a new standpoint:

The two Kerker conditions for a magnetodielectric dipolar particle are those at which the total reactive power is near (a)

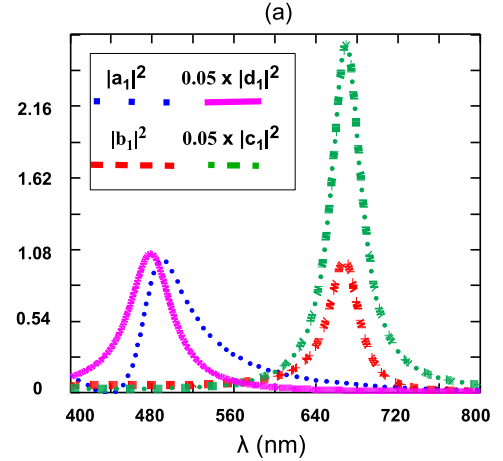

(b)

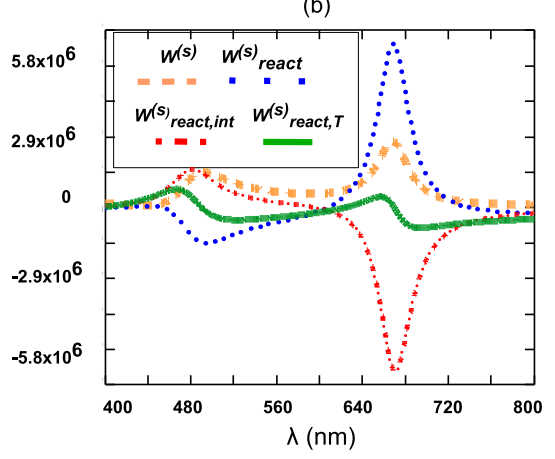

(c)

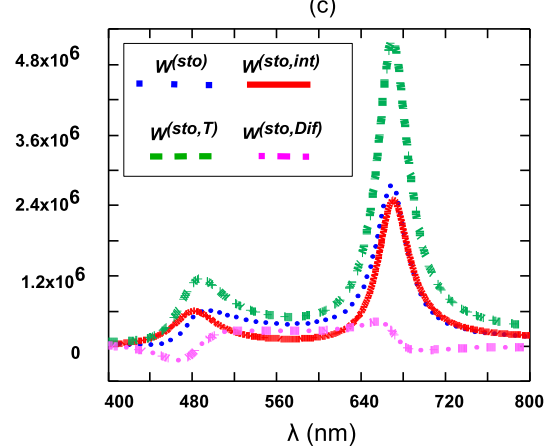

FIG. 4. (a) Si sphere of radius $a=75 \mathrm{~nm}$. (a) Square moduli of the electric and magnetic Mie coefficients, $a_{1}$ and $b_{1}$, and $d_{1}$ and $c_{1}$ of the external and interior scattered field, respectively. (b) Scattered power $W^{(s)}$ and reactive powers: external $W_{\text {react }}^{(s)}$, interior $W_{\text {react, int }}^{(s)}$, and total $W_{\text {react }, T}^{(s)}=W_{\text {react }}^{(s)}+W_{\text {react,int }}^{(s)}$. Notice that $W_{\text {react }, T}^{(s)}$ vanishes at wavelenghts close to those of the electric and magnetic Mie resonances: $\lambda_{e}=492$ $\mathrm{nm}$ and $\lambda_{m}=668 \mathrm{~nm}$, where $W^{(s)}$ is maximum. The lines $\left|a_{1}\right|^{2}$ and $\left|b_{1}\right|^{2}$ cross each other at the Kerker wavelengths: $\lambda_{K 1}=738.5 \mathrm{~nm}[$ at which $W_{\text {react }}^{(s)}=0$ and $\left.W_{\text {react, int }}^{(s)} \simeq 0\right]$ and $\lambda_{K 2}=608 \mathrm{~nm}$ [where both $W_{\text {react }}^{(s)}$ and $W_{\text {react,int }}^{(s)}$ are near 0]. (c) Stored energies: external $W^{(\text {sto })}$, interior $W^{(\text {sto,int) }}$, total $W^{\text {(sto, } T)}=W^{\text {(sto) }}+W^{\text {(sto,int) }}$, and difference $W^{\text {(sto,Dif) }}=W^{\text {(sto) }}-W^{\text {(sto,int) }}$. 
(a)

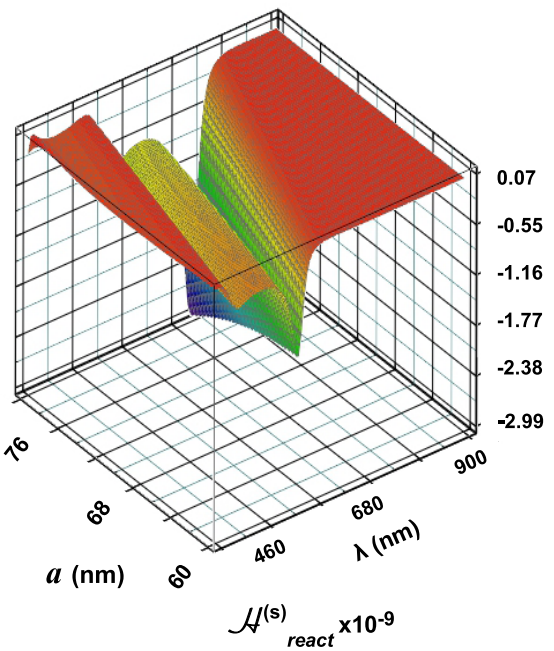

(b)

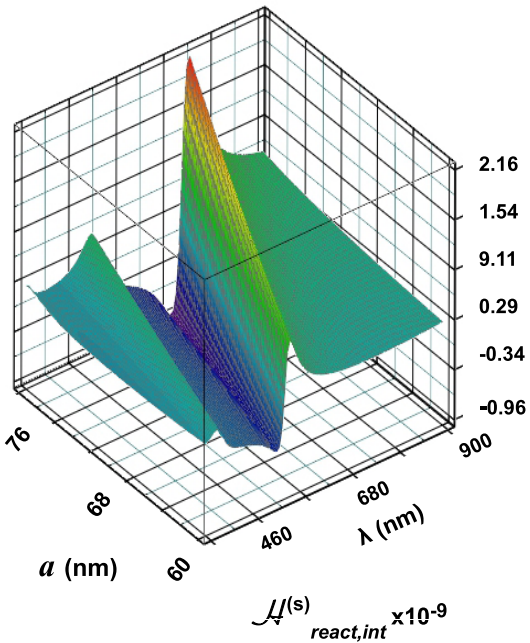

(c)

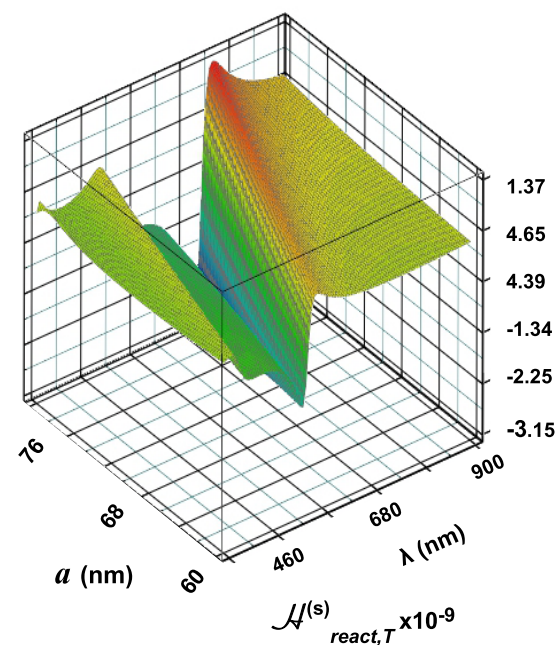

FIG. 5. 3D graphs of reactive helicities as functions of wavelength $\lambda$ and radius $a$ of a magnetodielelectric sphere of Si in air, illuminated by a left circularly polarized incident plane wave, $\operatorname{CPL}(+)$. (a) External $\mathcal{H}_{\text {react }}^{(s)}(\lambda, a)$. (b) Interior $\mathcal{H}_{\text {react,int }}^{(s)}(\lambda, a)$. (c) Total $\mathcal{H}_{\text {react }, T}^{(s)}(\lambda, a)=$ $\mathcal{H}_{\text {react }}^{(s)}(\lambda, a)+\mathcal{H}_{\text {react,int }}^{(s)}(\lambda, a)$. The two dips of $\mathcal{H}_{\text {react }}^{(s)}$ and zero crossings of $\mathcal{H}_{\text {react,int }}^{(s)}$ are close to the electric and magnetic resonant wavelengths $\lambda_{e}$ and $\lambda_{m}$ and are redshifted with increasing $a$. Irrespective of the value of $a, \mathcal{H}_{\text {react }}^{(s)}=0$ at $\lambda_{K 1}$ and $\mathcal{H}_{\text {react }, T}^{(s)}=0$ close to $\lambda_{e}$ and $\lambda_{m}$.

zero. Namely, the external reactive power $W_{\text {react }}^{(s)}$ is either zero (in $\mathrm{K} 1$ ), or close to zero (in $\mathrm{K} 2$ ); while the internal reactive power is near zero both in $\mathrm{K} 1$ and $\mathrm{K} 2$. In consequence, the reactive power underlies the angular distribution of scattered (or radiated) intensity and, hence, the directivity of the magnetoelectric particle in a way complementary to that formerly addressed in Mie-tronics and RF antennas [11,12].

\section{The reactive helicity}

Concerning the complex helicity flow of the fields scattered by the dipolar particle, [cf. Eq.(16)], $\mathcal{F}_{C}^{(s)}=\mathcal{F}^{(s)}+i \mathcal{F}_{\mathscr{H}_{\mathscr{R}}}^{(s)}=$ $(c / 4 k) \operatorname{Im}\left\{\left(\mathbf{B}^{(s) *} \times \mathbf{B}^{(s)}+\mathbf{E}^{(s) *} \times \mathbf{E}^{(s)}\right)+i\left(\mathbf{B}^{(s) *} \times \mathbf{B}^{(s)}-\right.\right.$ $\left.\left.\mathbf{E}^{(s) *} \times \mathbf{E}^{(s)}\right)\right\}$, we obtain the flux $\mathcal{F}_{C}^{(s)} \cdot \hat{\mathbf{r}}$ across the spherical surface $\partial V$ of radius $r \geqslant a$ centered in the particle. From Eqs. (D1) and (D2) for the fields in Appendix D, the terms that do not vanish on integration are

$$
\begin{aligned}
\int_{\partial V} \mathcal{F}_{C}^{(s)} \cdot \hat{\mathbf{r}} d^{2} r= & \frac{c}{4 k} r^{2} \int_{0}^{2 \pi} d \phi \int_{0}^{\pi} d \theta \hat{\mathbf{r}} \cdot \operatorname{Im}\left\{\left[\hat{\mathbf{r}} \times\left(\mathbf{m}^{*} \times \hat{\mathbf{r}}\right)\right]\right. \\
& \times(\hat{\mathbf{r}} \times \mathbf{p}) \frac{k^{2}}{r}\left(\frac{k^{2}}{r}+\frac{i k}{r^{2}}\right)-\text { c.c. } \\
& +\left[\hat{\mathbf{r}} \times\left(\mathbf{p}^{*} \times \hat{\mathbf{r}}\right)\right] \times(\hat{\mathbf{r}} \times \mathbf{m}) \frac{k^{2}}{r}\left(\frac{k^{2}}{r}+\frac{i k}{r^{2}}\right) \\
& - \text { c.c. }\},
\end{aligned}
$$

where c.c. denotes complex-conjugated of the previous term. A straightforward calculation of (36) yields

$$
\int_{\partial V} \mathcal{F}_{C}^{(s)} \cdot \hat{\mathbf{r}} d^{2} r=\frac{8 \pi}{3} c k^{2}\left\{k \operatorname{Im}\left[\mathbf{p} \cdot \mathbf{m}^{*}\right]+\frac{i}{r} \operatorname{Re}\left[\mathbf{p} \cdot \mathbf{m}^{*}\right]\right\},
$$

whose real part, $\mathcal{H}^{(s)}=\int_{\partial V} \operatorname{Re}\left\{\mathcal{F}_{C}^{(s)}\right\} \cdot \hat{\mathbf{r}} d^{2} r=\int_{\partial V} \mathcal{F}^{(s)}$. $\hat{\mathbf{r}} d^{2} r=\frac{8 \pi}{3} c k^{3} \operatorname{Im}\left[\mathbf{p} \cdot \mathbf{m}^{*}\right]$, is the total helicity of the scattered field (as such, it coincides with Eq. (25) of Ref. [17]). Like the scattered power, this helicity does not depend on the distance $r$.

However, the imaginary part of (37),

$$
\begin{aligned}
-\mathcal{H}_{\text {react }}^{(s)} & =\int_{\partial V} \operatorname{Im}\left\{\mathcal{F}_{C}^{(s)}\right\} \cdot \hat{\mathbf{r}} d^{2} r=\int_{\partial V} \mathcal{F}_{\mathscr{H}_{\mathscr{R}}}^{(s)} \cdot \hat{\mathbf{r}} d^{2} r \\
& =\frac{8 \pi}{3 r} c k^{2} \operatorname{Re}\left[\mathbf{p} \cdot \mathbf{m}^{*}\right],
\end{aligned}
$$

comes from the interference of the intermediate field with $r^{-2}$ dependence and the far field. $\mathcal{H}_{\text {react }}^{(s)}$ represents the external reactive helicity,

$$
\mathcal{H}_{\text {react }}^{(s)}=2 \omega \int_{V_{\infty}-V} \mathscr{H}_{\mathscr{R}}^{(s)} d^{3} r
$$

outside $V$, as detailed in its optical theorem discussed later, [cf. Eq. (52)]. It decreases as $r^{-1}$ as $r$ grows. Therefore, in analogy with the reactive power, in the near field $(r \ll \lambda)$, the reactive helicity dominates upon $\mathcal{H}^{(s)}$. On making $V=V_{0}$, $r=a$, Eq. (39) becomes the overall reactive helicity outside the particle.

It is of interest to specify the overall reactive helicity of the field inside the particle, $\mathcal{H}_{\text {react,int }}^{(s)}$, which is obtained integrating in $V_{0}$ the helicity of the internal field: $2 \omega \int_{V_{0}} \mathscr{H}_{\mathscr{R}}^{(s, \text { int })} d^{3} r$. For a left circularly polarized incident plane wave of unit intensity, this quantity is equal to $\left(8 \pi c k^{2} / 3 a\right)\left(9 / 4 k^{6}\right) 0.234 \times$ $0.276 \operatorname{Re}\left\{d_{1} c_{1}^{*}\right\}$.

Figures 5 illustrate $\mathcal{H}_{\text {react }}^{(s)}(\lambda, a), \quad \mathcal{H}_{\text {react,int }}^{(s)}(\lambda, a)$, and $\mathcal{H}_{\text {react }, T}^{(s)}(\lambda, a)=\mathcal{H}_{\text {react }}^{(s)}(\lambda, a)+\mathcal{H}_{\text {react,int }}^{(s)}(\lambda, a)$, generated by Si spheres illuminated by a left circularly polarized, $\mathrm{CPL}(+)$, plane wave. Again, the redshift with increasing radius $a$ is observed in the electric and magnetic dipole resonant dips of 
(a)

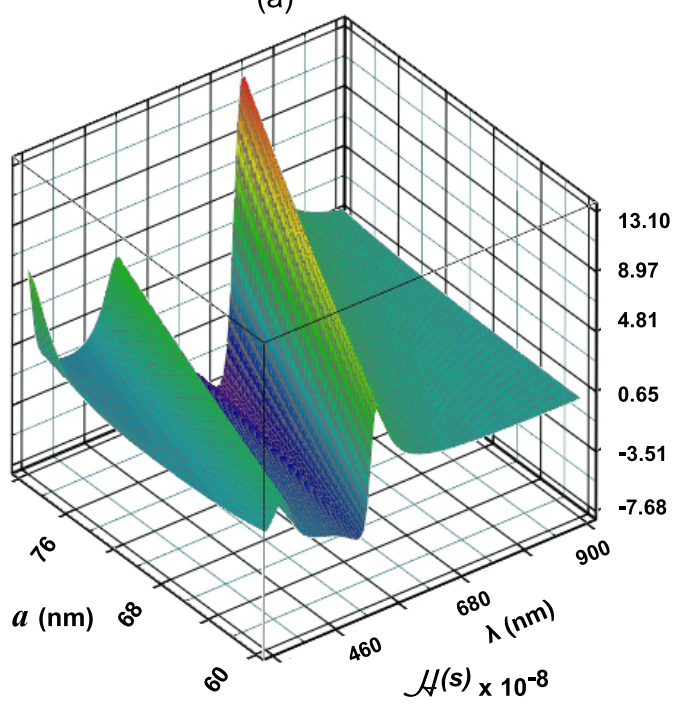

(b)

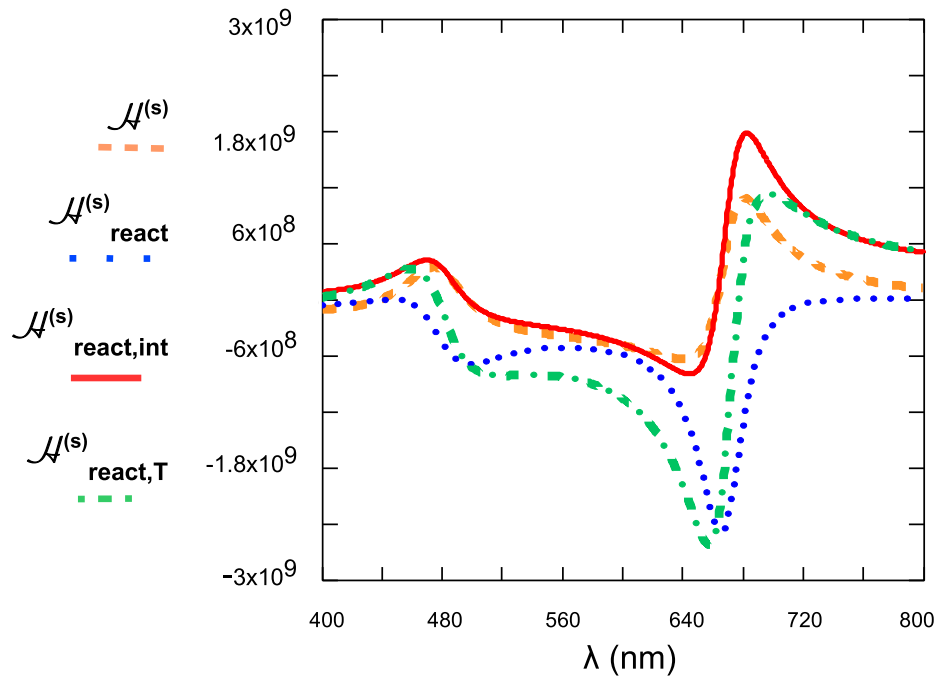

FIG. 6. (a) 3D graph of the scattered helicity $\mathcal{H}^{(s)}(\lambda, a)$ generated by Si spheres in air, illuminated by a left circularly polarized incident plane wave, CPL(+). It has two peaks, at 475 and $679 \mathrm{~nm}$, influenced by the electric and magnetic resonant wavelengths $\lambda_{e}=492 \mathrm{~nm}$ and $\lambda_{m}=668 \mathrm{~nm}$. (b) Making $a=75 \mathrm{~nm}, \mathcal{H}^{(s)}(\lambda), \mathcal{H}_{\text {react }}^{(s)}(\lambda), \mathcal{H}_{\text {react, int }}^{(s)}(\lambda)$, and $\mathcal{H}_{\text {react }, T}^{(s)}(\lambda)$. At the Kerker wavelength $\lambda_{K 1}=738.5 \mathrm{~nm}, \mathcal{H}_{\text {react }}^{(s)}$ vanishes. Also $\mathcal{H}_{\text {react, } T}^{(s)}(\lambda)$ is zero close to the wavelengths where the scattered helicity $\mathcal{H}^{(s)}$ is maximum, and in the vicinity of extrema of both $\mathcal{H}_{\text {react }}^{(s)}$ and $\mathcal{H}_{\text {react,int }}^{(s)}$.

$\mathcal{H}_{\text {react }}^{(s)}$. An interesting feature of $\mathcal{H}_{\text {react, int }}^{(s)}$ is its similitude with $\mathcal{H}^{(s)}$, [compare Fig. 5(b) with Fig. 6(a) as well as $\mathcal{H}_{\text {react, int }}^{(s)}$ with $\mathcal{H}^{(s)}$ in Fig. 6(b)], and specially their similarity with the converted helicity in the range between $\lambda_{e}$ and $\lambda_{m}$ (compare with Fig. 1(c) of Ref. [56]). This remarks the contribution of the interior reactive helicity to the scattered helicity line shape and lays down an intriguing connection with previous studies [54-56] which attribute the conversion of helicity to contributions of the scatterer volume and surface.

The total reactive helicity $\mathcal{H}_{\text {react, } T}^{(s)}$ plays on the scattered helicity a role analogous to that of the total reactive power on the scattered power. As seen in Fig. 6(a), $\mathcal{H}^{(s)}$ has resonant dips influenced by the electric and magnetic resonant wavelengths, $\lambda_{e}$ and $\lambda_{m}$, of the scattered power. For $a=75$ [cf. Fig. 6(b)], these dips are at $\lambda=475$ and $679 \mathrm{~nm}$ and stem from the resonances at $\lambda_{e}=492 \mathrm{~nm}$ and $\lambda_{m}=668 \mathrm{~nm}$, respectively. On the other hand, $\mathcal{H}_{\text {react }, T}^{(s)}$ becomes zero very close to these resonant wavelengths of $\mathcal{H}^{(s)}$. We conclude, therefore, that the interior reactive helicity $\mathcal{H}_{\text {react,int }}^{(s)}$ counteracts on the external reactive helicity $\mathcal{H}_{\text {react }}^{(s)}$ close the resonant wavelengths of $\mathcal{H}^{(s)}$ (where also $\mathcal{H}_{\text {react }}^{(s)}$ and $\mathcal{H}_{\text {react,int }}^{(s)}$ are near extreme values), yielding a zero total reactive helicity $\mathcal{H}_{\text {react, } T}^{(s)}$. This property may be observed in Fig. 5(b), independently of $a$, and it is clearly seen in Fig. 6(b). We do not know, however, of any analogy of these effects in RF-antenna theory.

Notice that these high index magnetoelectric particles have the interesting property of emitting a wavefield in which there are not very large peaks of the total reactive power [cf. Fig. 4(b)] versus those of the scattered (radiated) power; although, certainly, where this reactive power has extrema the scattered (radiated) power is well aside its peaks. However, under chiral illumination these fields present high peaks of total reactive helicity versus its radiated one. For instance, [see in Fig. 6(b)], the 75-nm particle] yields a the total reactive helicity with a large dip at $658 \mathrm{~nm}$ where the scattered (radiated) helicity is near its minimum value. This occurs at shifted positions as $a$ varies, [cf. Figs. 6(a) and 5(b)].

Therefore the picture that emerges in these illustrations of such optical nanoantennas, considered as either primary or secondary sources, is that the reactive power and the reactive helicity, which are concentrated both inside and in the near and intermediate regions of the source, have a hampering effect in their far field scattering (or radiation) efficiency. This is an analogous effect to that due to the presence of reactive power in RF antennas. In consequence, if these nanoantennas emit chiral light, the total reactive helicity hinders the efficiency of far field scattered (or radiated) helicity, so that less of this helicity is emitted due to a build-up of reactive helicity in and around the nanoantenna [67].

Under incident CPL these particles are dual at $\lambda_{K 1}$, and then $\mathbf{p}_{ \pm}= \pm i \mathbf{m}_{ \pm}[16,17]$, the upper and lower sign applying to left circular, $\mathrm{CPL}(+)$ and right circular, $\mathrm{CPL}(-)$, respectively. Then $\mathbf{p} \cdot \mathbf{m}^{*}$ is purely imaginary at $\lambda_{K 1}$ [68], and one sees from (37) that $\mathcal{H}_{\text {react }}^{(s)}=0$. This is observed in Fig. 5(a) for any $a$ and in Fig. 6(b) at $\lambda_{K 1}=738.5 \mathrm{~nm}$.

As $a$ varies, $\mathcal{H}_{\text {react }}^{(s)}(\lambda, a)$ vanishes at the corresponding Kerker wavelength $\lambda_{K 1}$; this is seen in detail in Fig. 6(b) for $a=75 \mathrm{~nm}$.

Therefore the first Kerker condition, K1, also has the novel property that under CPL illumination, the magnetodielectric particle, which then becomes dual and hence emits a wavefield of well-defined helicity equal to the incident one $[16,17,56]$, does not generate external reactive helicity. 


\section{THE REACTIVE POWER OPTICAL THEOREM}

Consider a wavefield $\mathbf{E}^{(i)}, \mathbf{H}^{(i)}$ incident on a magnetodielectric body of volume $V_{0}$. The field at any point of the embedding medium (assumed to be vacuum or air) is represented as $\mathbf{E}=\mathbf{E}^{(i)}+\mathbf{E}^{(s)}, \mathbf{H}=\mathbf{H}^{(i)}+\mathbf{H}^{(s)}$, where the superscript $(s)$ denotes the scattered field. Maxwell's equations are written as

$$
\begin{aligned}
& \nabla \times \mathbf{H}^{(i)}=-i k \mathbf{D}^{(i)}, \nabla \times \mathbf{E}^{(i)}=i k \mathbf{B}^{(i)} ; \\
& \nabla \times \mathbf{H}^{(s)}=-i k \mathbf{E}^{(s)}-4 \pi i k \mathbf{P}+\frac{4 \pi}{c} \mathbf{j}, \\
& \nabla \times \mathbf{E}^{(s)}=i k \mathbf{H}^{(s)}+4 \pi i k \mathbf{M} .
\end{aligned}
$$

Where $\mathbf{P}, \mathbf{M}$, and $\mathbf{j}$ are the polarization, magnetization, and free current densities in $V_{0}$, respectively. $\mathbf{D}^{(i)}=\mathbf{E}^{(i)}, \mathbf{B}^{(i)}=$ $\mathbf{H}^{(i)}$. We insert Eqs. (40) into Eq. (1) for the total fields $\mathbf{E}$ and $\mathbf{H}$ and use the identity: $\nabla \cdot\left(\mathbf{E} \times \mathbf{H}^{*}\right)=\mathbf{H}^{*} \cdot \nabla \times \mathbf{E}-\mathbf{E}$. $\nabla \times \mathbf{H}^{*}$, integrating in a volume $V$ that contains $V_{0}$,

$$
\begin{gathered}
\int_{V} d^{3} r\left\{2 i \omega\left(\left\langle w_{m}^{(i)}\right\rangle-\left\langle w_{e}^{(i)}\right\rangle\right)+i \frac{\omega}{4 \pi} \operatorname{Re}\left\{\mathbf{H}^{(i)} \cdot \mathbf{H}^{(s) *}\right.\right. \\
\left.-\mathbf{E}^{(i)} \cdot \mathbf{E}^{(s) *}\right\}+i \frac{\omega}{2}\left[\mathbf{H}^{(i) *} \cdot \mathbf{M}-\mathbf{E}^{(i)} \cdot \mathbf{P}^{*}\right] \\
\left.-\frac{1}{2} \mathbf{j}^{*} \cdot \mathbf{E}^{(i)}+\nabla \cdot \mathbf{S}^{(s)}\right\} d^{3} r \\
=-\frac{1}{2} \int_{V_{0}} d^{3} r \mathbf{j}^{*} \cdot \mathbf{E}+2 i \omega \int_{V}\left(\left\langle w_{m}\right\rangle-\left\langle w_{e}\langle) d^{3} r .\right.\right.
\end{gathered}
$$

If the incident field has no evanescent components, i.e., it is source-free and propagating, it does not store energy, so that the first term of (41) is identically zero. However if it is evanescent, or it has evanescent components, we shaw in (28) that it stores reactive power and it is given by this term. Therefore we shall keep it in the above equation, which is the complex optical theorem in presence of the scatterer.

If there are no scattering induced sources other than $\mathbf{P}$ and $\mathbf{M}$ in the body, the free current $\mathbf{j}$ conveys the conversion of incident power into mechanical and/or thermal energy through the work done on the charges $\frac{1}{2} \operatorname{Re} \int_{V_{0}} d^{3} r \mathbf{j}^{*} \cdot(\mathbf{E}-$ $\mathbf{E}^{(i)}$ ), which accounts for the decrease of energy $\mathcal{W}^{(a)}$ from the wave as power absorbed by the body. Then taking the real part of (41), we obtain

$$
\begin{array}{r}
\frac{\omega}{2} \int_{V_{0}} d^{3} r \operatorname{Im}\left[\mathbf{H}^{(i) *} \cdot \mathbf{M}+\mathbf{E}^{(i) *} \cdot \mathbf{P}\right] \\
\quad=\mathcal{W}^{(a)}+\int_{\partial V} d^{2} r \operatorname{Re}\left\{\mathbf{S}^{(s)}\right\} \cdot \hat{\boldsymbol{r}}
\end{array}
$$

which is the standard optical theorem (OT) for energy [21,60], describing the extinction of incident energy [the left-hand side of (42)], and consequent absorption and radiation of the total scattered energy. It reduces to its well-known expression [21] for dipolar particles on making $\mathbf{P}(\mathbf{r})=\mathbf{p} \delta(\mathbf{r})$ and $\mathbf{M}(\mathbf{r})=$ $\mathbf{m} \delta(\mathbf{r})$, and then $\int_{\partial V} d^{2} r \operatorname{Re}\left\{\mathbf{S}^{(s)}\right\} \cdot \hat{\boldsymbol{r}}=W^{(s)}$ in accordance with (32). (41),

Here we are, however, interested in the imaginary part of

$$
\begin{aligned}
\int_{V} d^{3} r\left[2 \omega\left(\left\langle w_{m}^{(i)}\right\rangle-\left\langle w_{e}^{(i)}\right\rangle\right)+\frac{\omega}{4 \pi} \operatorname{Re}\left\{\mathbf{H}^{(i)} \cdot \mathbf{H}^{(s) *}-\mathbf{E}^{(i)} \cdot \mathbf{E}^{(s) *}\right\}+\frac{\omega}{2} \operatorname{Re}\left\{\mathbf{H}^{(i) *} \cdot \mathbf{M}-\mathbf{E}^{(i) *} \cdot \mathbf{P}\right\}\right] \\
-\frac{1}{2} \operatorname{Im}\left\{\int_{V_{0}} d^{3} r \mathbf{j}^{*} \cdot \mathbf{E}^{(i)}\right\}+\int_{\partial V} \operatorname{Im}\left\{\mathbf{S}^{(s)}\right\} \cdot \hat{\boldsymbol{r}} d^{2} r \\
=-\frac{1}{2} \int_{V_{0}} d^{3} r \operatorname{Im}\left\{\mathbf{j}^{*} \cdot \mathbf{E}\right\}+2 \omega \int_{V}\left(\left\langle w_{m}\right\rangle-\left\langle w_{e}\right\rangle\right) d^{3} r,
\end{aligned}
$$

which becomes

$$
\frac{\omega}{2} \int_{V_{0}} d^{3} r \operatorname{Re}\left\{\mathbf{H}^{(i) *} \cdot \mathbf{M}-\mathbf{E}^{(i) *} \cdot \mathbf{P}\right\}=-\frac{1}{2} \int_{V_{0}} d^{3} r \operatorname{Im}\left\{\mathbf{j}^{*} \cdot \mathbf{E}^{(s)}\right\}-\int_{\partial V} \operatorname{Im}\left\{\mathbf{S}^{(s)}\right\} \cdot \hat{\boldsymbol{r}} d^{2} r+2 \omega \int_{V}\left(\left\langle\tilde{w}_{m}^{(s)}\right\rangle-\left\langle\tilde{w}_{e}^{(s)}\right\rangle\right) d^{3} r,
$$

where we have made use of the fact that $\left\langle w_{m}^{(s)(\mathrm{FF})}\right\rangle=\left\langle w_{e}^{(s)(\mathrm{FF})}\right\rangle$ and hence $\left\langle w_{m}^{(s)}\right\rangle-\left\langle w_{e}^{(s)}\right\rangle=\left\langle\tilde{w}_{m}^{(s)}\right\rangle-\left\langle\tilde{w}_{e}^{(s)}\right\rangle$ [cf. paragraph prior to Eq. (34)].

Equation (44) is our formulation of the reactive power optical theorem (ROT) for a generic scatterer whose response to illumination induces densities of polarization $\mathbf{P}$, magnetization $\mathbf{M}$, and free current $\mathbf{j}$ [69]. The left-hand side constitutes the extinction of incident energy which produces the build-up of external reactive power on scattering in the right-hand side of (44). Thus Eq. (44) describes how, in addition to being radiated into the far zone as Eq. (42) illustrates, scattering gives rise to nonradiated energy, stored in $V$ in the form of reactive power, flowing out from the scattering object and returning to it.
For a dipolar particle the ROT reduces to

$$
\begin{aligned}
\frac{\omega}{2} \operatorname{Re}\left\{\mathbf{H}^{(i) *}(0) \cdot \mathbf{m}-\mathbf{E}^{(i) *}(0) \cdot \mathbf{p}\right\} \\
=-\frac{1}{2} \int_{V_{0}} d^{3} r \operatorname{Im}\left\{\mathbf{j}^{*} \cdot \mathbf{E}^{(s)}\right\}-\int_{\partial V} \operatorname{Im}\left\{\mathbf{S}^{(s)}\right\} \cdot \hat{\boldsymbol{r}} d^{2} r \\
\quad+2 \omega \int_{V}\left(\left\langle\tilde{w}_{m}^{(s)}\right\rangle-\left\langle\tilde{w}_{e}^{(s)}\right\rangle\right) d^{3} r .
\end{aligned}
$$

The argument 0 indicates that the fields are evaluated at the particle center $r=0$.

Concerning a dipolar particle, the process described by the complex optical theorem (41) is analogous to that in which the feeding energy from an alternate current $I$, induces an oscillating dipole in a small antenna, which emits radiated and stored 
power through the extinction, $\frac{1}{2} Z I^{2}$, of the driving energy. $Z$ being the antenna input impedance, $Z=R_{l}+R_{r}-i X$. The dipole loss resistance $R_{l}$ and radiation resistance $R_{r}$ [12], generated in accordance with the optical theorem (42), are $2 W^{(a)} /|I|^{2}$ and $2 W^{(s)} /|I|^{2}$, respectively. On the other hand, the dipole reactance $X$ (which for a magnetoelectic dipole is either capacitive or inductive [9], depending on the wavelength $\lambda_{e}$ or $\left.\lambda_{m}\right)$ stems from its external reactive power, $W_{\text {react }}^{(s)}$ [12], whose generation is ruled by the ROT (45). Hence, in this context, the extinction term in the left-hand sides of (42) and (45) may be associated to $\frac{1}{2}\left(R_{l}+R_{r}\right)|I|^{2}$ and $-\frac{1}{2} X|I|^{2}$, respectively.

On taking $\partial V$ and $V$ as $\partial V_{\infty}$ and $V_{\infty}$, respectively, corresponding to a large sphere, $(k r \rightarrow \infty)$, the flux of scattered CPV across $\partial V_{\infty}$ is real and equals the total scattered energy $W^{(s)}$. Therefore Eq. (45) yields

$$
\begin{aligned}
\frac{\omega}{2} \operatorname{Re} & \left\{\mathbf{H}^{(i) *}(0) \cdot \mathbf{m}-\mathbf{E}^{(i) *}(0) \cdot \mathbf{p}\right\} \\
= & -\frac{1}{2} \int_{V_{0}} d^{3} r \operatorname{Im}\left\{\mathbf{j}^{*} \cdot \mathbf{E}^{(s)}\right\} \\
& +2 \omega \int_{V_{\infty}}\left(\left\langle\tilde{w}_{m}^{(s)}\right\rangle-\left\langle\tilde{w}_{e}^{(s)}\right\rangle\right) d^{3} r,
\end{aligned}
$$

which introduced into (45) leads to

$$
\begin{aligned}
& -\int_{\partial V} \operatorname{Im}\left\{\mathbf{S}^{(s)}\right\} \cdot \hat{\boldsymbol{r}} d^{2} r \\
& =2 \omega \int_{V_{\infty}-V}\left(\left\langle\tilde{w}_{m}^{(s)}\right\rangle-\left\langle\tilde{w}_{e}^{(s)}\right\rangle\right) d^{3} r=W_{\text {react }}^{(s)}
\end{aligned}
$$

In contrast with its real part, the flow (47) depends on the integration domains, $V$ and $\partial V$. Notice that (47) coincides with Eq. (34).

\section{THE REACTIVE HELICITY OPTICAL THEOREM}

Next, we put forward the law which rules the formation of external reactive helicity by scattering in the near and intermediate field regions of the particle through extinction of helicity of the incident wave. Let us introduce Eqs. (40) into the identities: $\nabla \cdot\left(\mathbf{H}^{*} \times \mathbf{H}\right)=\mathbf{H} \cdot \boldsymbol{\nabla} \times \mathbf{H}^{*}-\mathbf{H}^{*} \cdot \boldsymbol{\nabla} \times$ $\mathbf{H}$ and $\nabla \cdot\left(\mathbf{E}^{*} \times \mathbf{E}\right)=\mathbf{E} \cdot \nabla \times \mathbf{E}^{*}-\mathbf{E}^{*} \cdot \nabla \times \mathbf{E}$, integrating in a volume $V$ that contains the scattering volume $V_{0}$. With $\mathbf{E}=\mathbf{E}^{(i)}+\mathbf{E}^{(s)}, \mathbf{H}=\mathbf{H}^{(i)}+\mathbf{H}^{(s)}$, adding the respective expressions, employing the conservation equation (12) and using the definitions (8) and (13) $\left(n=1\right.$ and $\mathbf{H}=\mathbf{B}$ outside $\left.V_{0}\right)$, we arrive at

$$
\begin{aligned}
& 2 \pi c \int_{V_{0}} d^{3} r \operatorname{Re}\left\{\mathbf{E}^{(i) *} \cdot \mathbf{M}-\mathbf{H}^{(i) *} \cdot \mathbf{P}\right\} \\
& \quad=\int_{\partial V} d^{2} r \mathcal{F}^{(s)} \cdot \mathbf{n}+\frac{2 \pi}{k} \int_{V_{0}} d^{3} r \operatorname{Im}\left\{\mathbf{H}^{(s) *} \cdot \mathbf{j}\right\},
\end{aligned}
$$

which is the known optical theorem for the electromagnetic helicity [17] applying to magnetodielectric arbitrary scattering bodies.

Notice that for a dipolar particle, since $\mathbf{P}(\mathbf{r})=\mathbf{p} \delta(\mathbf{r})$, $\mathbf{M}(\mathbf{r})=\mathbf{m} \delta(\mathbf{r})$ and $\int_{\partial V} d^{2} r \mathcal{F}^{(s)} \cdot \mathbf{n}=\left(8 \pi c k^{3} / 3\right) \operatorname{Im}\left[\mathbf{p} \cdot \mathbf{m}^{*}\right]$, Eq. (48) becomes like Eq. (28) of Ref. [17].
However, our focus is the conservation law of the reactive helicity. To formulate it in the form of an optical theorem we subtract, rather than add, the above vector identities, and make use of the conservation law (14) along with definitions (9) and (15). Then, proceeding as before, it is straightforward to obtain

$$
\begin{aligned}
2 \pi c & \int_{V_{0}} d^{3} r \operatorname{Re}\left\{\mathbf{E}^{(i) *} \cdot \mathbf{M}+\mathbf{H}^{(i) *} \cdot \mathbf{P}\right\} \\
= & -\frac{2 \pi}{k} \int_{V_{0}} d^{3} r \operatorname{Im}\left\{\mathbf{H}^{(s) *} \cdot \mathbf{j}\right\}-\int_{\partial V} d^{2} r \mathcal{F}_{\mathscr{H}_{\mathscr{R}}^{(s)}} \cdot \mathbf{n} \\
& +2 \omega \int_{V} d^{3} r \mathscr{H}_{\mathscr{R}}^{(s)} .
\end{aligned}
$$

Equation (49) is the reactive helicity optical theorem and applies to a generic magnetodielectric scatterer [70]. The lefthand side represents the extinction of helicity of the incident wave on build-up outside the body of a reactive helicity by scattering, given by the right-hand side of (49). Thus, like the energy, the incident helicity gives rise to a reactive one associated to the scattered field, which, in addition to the internal reactive helicity, is stored around the particle, dominating in the near and intermediate field regions where it flows back and forth from the scatterer.

This storage is seen by first considering $V$ to be $V_{\infty}$ in (49). Then (cf. Ref. [71])

$$
\int_{\partial V_{\infty}} d^{2} r \mathcal{F}_{\mathscr{H}_{\mathscr{R}}}^{(s)} \cdot \mathbf{n}=0 .
$$

Therefore

$$
\begin{aligned}
& 2 \pi c \int_{V_{0}} d^{3} r \operatorname{Re}\left\{\mathbf{E}^{(i) *} \cdot \mathbf{M}+\mathbf{H}^{(i) *} \cdot \mathbf{P}\right\} \\
& \quad=-\frac{2 \pi}{k} \int_{V_{0}} d^{3} r \operatorname{Im}\left\{\mathbf{H}^{(s) *} \cdot \mathbf{j}\right\}+2 \omega \int_{V_{\infty}} d^{3} r \mathscr{H}_{\mathscr{R}}^{(s)},
\end{aligned}
$$

which substituted in (49) leads to

$$
-\int_{\partial V} d^{2} r \mathcal{F}_{\mathscr{H}_{\mathscr{R}}}^{(s)} \cdot \mathbf{n}=2 \omega \int_{V_{\infty}-V} d^{3} r \mathscr{H}_{\mathscr{R}}^{(s)} .
$$

Notice that if $V=V_{0}$, Eq. (52) accounts for the reactive helicity stored outside the scattering body.

Likewise, if the scatterer is a dipolar particle, the extinction term in the left-hand side of (51) becomes $2 \pi c \operatorname{Re}\left\{\mathbf{E}^{(i) *}(0)\right.$.

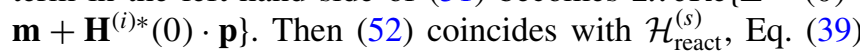
according to (38), thus proving it; and illustrates how the external reactive helicity is stored around the particle without being scattered into the far zone.

\section{CONSEQUENCE OF THE REACTIVE POWER OPTICAL THEOREM: SIGNIFICANCE OF THE REACTIVE HELICITY IN REACTIVE DICHROISM}

In dichroism, chiral light illuminates a chiral particle, molecule, or nanostructure. Assuming it dipolar, its constitutive relations for the induced dipole moments, $\mathbf{p}$ and $\mathbf{m}$, and the incident field are

$$
\mathbf{p}=\alpha_{e} \mathbf{E}^{(i)}+\alpha_{\mathrm{em}} \mathbf{B}^{(i)}, \mathbf{m}=\alpha_{m e} \mathbf{E}^{(i)}+\alpha_{m} \mathbf{B}^{(i)} .
$$


The electric, magnetic, and magnetoelectric polarizabilities being $\alpha_{e}, \alpha_{m}, \alpha_{\mathrm{em}}$, and $\alpha_{m e}$; and fulfilling $\alpha_{\mathrm{em}}=-\alpha_{m e}$ since the object is chiral $[14,72]$.

The signal re-emitted (or scattered) by the excitation of this dipolar body discriminates enantiomers (i.e., particles with either $\alpha_{m e}$ or $\left.-\alpha_{m e}\right)[14,72,73]$ by using as rate of excitation: $\mathcal{W}^{(s)}=\frac{\omega}{2} \operatorname{Im}\left\{\mathbf{E}^{(i) *}(0) \cdot \mathbf{p}+\mathbf{B}^{(i) *}(0) \cdot \mathbf{m}\right\}$ [cf. left-hand side of Eq. (42)].

For instance, consider the pair of illuminating fields [14]: $\mathcal{E}^{(i)}(\mathbf{r}, t)=\operatorname{Re}\left[ \pm \mathbf{E}^{(i)}(\mathbf{r}) \exp (-i \omega t)\right]$ and $\mathcal{H}^{(i)}(\mathbf{r}, t)=$ $\operatorname{Re}\left[\mathbf{H}^{(i)}(\mathbf{r}) \exp (-i \omega t)\right]$, whose respective helicities are $\mathscr{H}_{i}^{+}$and $\mathscr{H}_{i}^{-}$, with $\mathscr{H}_{i}^{+}=\mathscr{H}_{i}=-\mathscr{H}_{i}^{-}$. On employing (42) and (53), the particle excitation rate becomes: $\mathcal{W}^{(s) \pm}=$ $\frac{\omega}{2}\left\{\alpha_{e}^{I}\left|\mathbf{E}^{(i)}(0)\right|^{2}+\alpha_{m}^{I}\left|\mathbf{B}^{(i)}(0)\right|^{2} \pm 2 \alpha_{m e}^{R} \operatorname{Im}\left[\mathbf{E}^{(i)} \cdot \mathbf{B}^{(i) *}\right]\right\}=\frac{\omega}{2}\left\{\alpha_{e}^{I}\right.$ $\left.\left|\mathbf{E}^{(i)}(0)\right|^{2}+\alpha_{m}^{I}\left|\mathbf{B}^{(i)}(0)\right|^{2} \pm 4 k \alpha_{m e}^{R} \mathscr{H}_{i}\right\}$. The superscripts $I$ and $R$ denote the imaginary and real parts, respectively. Clearly, the sign + or - appears according to whether the helicity of the illumination is positive $\mathscr{H}_{i}^{+}=\mathscr{H}_{i}$ or negative $\mathscr{H}_{i}^{-}=-\mathscr{H}_{i}$. Then the above expression of $\mathcal{W}^{(s) \pm}$ yields the well-known dissymmetry factor $g=2\left(\mathcal{W}^{(s)+}-\mathcal{W}^{(s)-}\right) /\left(\mathcal{W}^{(s)+}+\mathcal{W}^{(s)-}\right)[72,73]$ proportional to $\alpha_{m e}^{R} \mathscr{H}_{i}[14]$.

However, the ROT establishes that rather than the power radiated in the far zone (42), one may address the excitation of stored reactive power, which dominates in the near and intermediate field regions of the particle, which is given by the left-hand side of Eq. (45), viz. $\mathcal{W}_{\text {react }}^{(s)}=\frac{\omega}{6} \Re\left\{\mathbf{E}^{(i) *}(0) \cdot \mathbf{p}-\right.$

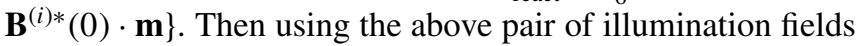
and Eq. (53), one obtains the discriminatory reactive power

$$
\begin{aligned}
\mathcal{W}_{\text {react }}^{(s) \pm} & =\frac{\omega}{2} \Re\left\{ \pm \mathbf{E}^{(i) *}(0) \cdot \mathbf{p}-\mathbf{B}^{(i) *}(0) \cdot \mathbf{m}\right\} \\
& =\frac{\omega}{2}\left\{\alpha_{e}^{R}\left|\mathbf{E}^{(i)}\right|^{2}-\alpha_{m}^{R}\left|\mathbf{B}^{(i)}\right|^{2} \mp 2 \alpha_{m e}^{R} \Re\left[\mathbf{E}^{(i)} \cdot \mathbf{B}^{(i) *}\right]\right\} \\
& =\frac{\omega}{2}\left\{\alpha_{e}^{R}\left|\mathbf{E}^{(i)}\right|^{2}-\alpha_{m}^{R}\left|\mathbf{B}^{(i)}\right|^{2} \mp 4 k \alpha_{m e}^{R} \mathscr{H}_{\mathscr{R} i}\right\}
\end{aligned}
$$

So that now the sign - or + applies according to whether the reactive helicity of the illumination is positive $\mathscr{H}_{\mathscr{R}}^{+}=\mathscr{H}_{\mathscr{R} i}$ or negative $\mathscr{H}_{\mathscr{R}_{i}}{ }^{-}=-\mathscr{H}_{\mathscr{R} i}$, respectively; and (54) yields a dissymmetry factor proportional to $-\alpha_{m e}^{R} \mathscr{H}_{\mathscr{R} i}[74]$.

We propose Eq. (54) as the basis of reactive dichroism observations. At difference with standard dichroism, it involves a chiral incident field with nonzero reactive helicity, and constitutes a near field optics technique.

Therefore, in an analogous way as detecting the radiated energy $\mathcal{W}^{(s) \pm}$ (or absorption/extinction energy), in standard dichroism involves the helicity $\mathscr{H}_{i}$ of the incident wave, in reactive dichroism, observing the excitation of reactive power $\mathcal{W}_{\text {react }}^{(s) \pm}$ in the chiral particle conveys the incident field reactive helicity $\mathscr{H}_{\mathscr{R} i}$, which evidently comes out on using (54) in a dissymmetry factor defined as $g_{\text {react }}=2\left(\mathcal{W}_{\text {react }}^{(s)+}-\right.$ $\left.\mathcal{W}_{\text {react }}^{(s)-}\right) /\left(\mathcal{W}_{\text {react }}^{(s)+}+\mathcal{W}_{\text {react }}^{(s)-}\right)$. Notice that being $\mathscr{H}_{\mathscr{R} i}$ measurable, so is $g_{\text {react }}$ in proportion to $\mathscr{H}_{\mathscr{R}}$.

There is a variety of pairs of illumination wavefields that, like in the above illustration, are interchangeable by parity, and that one may employ in experiments. As discussed in previous sections, nonpropagating fields in free-space possess a nonzero $\mathscr{H}_{\mathscr{R} i}$; e.g., elliptically (or circularly, in particu- lar) polarized standing waves, near fields from an emitter, or evanescent and other surface waves, fulfill Eq. (54).

\section{CONCLUSIONS}

Given the broad interest of evanescent waves at the nanoscale [75] and of small particles as light emitting nanoantennas, couplers, and metasurfaces elements, the contributions of this paper on its reactive quantities is summarized in the following main conclusions.

(1) We have established the concepts of complex helicity density and its complex helicity flow, together with their conservation law that we name complex helicity theorem. Its real part is the well-known conservation equation of optical helicity, while its imaginary is a novel law that governs the build-up of reactive helicity through its imaginary, and thus zero timeaverage, flow. The concept of reactive helicity density unifies that of magnetoelectric energy density, previously introduced by symmetry arguments, and the so-called real helicity. In this way, we put forward its conservation law and observability, thus completing the fundamentals of this quantity.

(2) The conservation of reactive helicity and reactive power, and their zero time-averaged flow, has been illustrated in two paradigmatic systems: an evanescent wave and a wavefield scattered (or emitted) by a dipolar magnetodielectric particle. For the former, we have put forward reactive orbital and spin momenta that characterize its imaginary (reactive) field (Poynting) momentum; showing that the wave density of reactive helicity is observable from an experimentally detectable mixed electric-magnetic transversal optical force exerted by the evanescent wave on a small high refractive index particle (which behaves as magnetodielectric) through this reactive Poynting momentum. On the other hand, we have uncovered a novel nonconservative force in the decay direction of the evanescent wave, which can be discriminated from the gradient one and thus detected, making observable the wavefield reactive power density.

(3) Concerning the stored energy, reactive power, and reactive helicity of the field scattered by a dipolar magnetodielectric particle, we have shown that they provide a novel framework to study the particle emission directivity. This has been illustrated on addressing the two Kerker conditions, K1 of zero backscattering, and $\mathrm{K} 2$ of minimum forward scattering. We have established that under CPL incident light, the external reactive power is zero in $\mathrm{K} 1$ or close to zero in $\mathrm{K} 2$; while the internal reactive power, and hence the total reactive power, is near zero both in $\mathrm{K} 1$ and $\mathrm{K} 2$. Also, we have proven an additional novel property of the particle at $\mathrm{K} 1$ wavelengths, namely, it produces a scattered field with nule overall external reactive helicity.

(4) We have established a reactive helicity optical theorem that governs the build-up and storage of near field reactive helicity, on extinction of the incident helicity as light interacts with a generally magnetodielectric nanoantenna. Also we have shown that the emission of resonant scattered power and of resonant scattered helicity coincides with a nule, or near zero, total reactive power and helicity, respectively, i.e., those given by the sum of the overall interior and external reactive powers and helicities. Conversely, peaks of total reactive 
helicity (reactive power) are associated with poorer efficiency in the emission of radiated (or scattered) helicity (power).

(5) A reactive power optical theorem has been put forward. It rules the formation of external reactive power and stored electric and magnetic energies, which dominate in the near and intermediate field zones of a magnetodielectric particle by extinction of the illuminating energy.

(6) This latter theorem provides a framework to studying the near field response of chiral nanoparticles to illumination with chiral complex fields. It is remarkable that in the phenomenon of dichroism on illumination with chiral light, the incident reactive helicity arises in the near field region, as we have shown, while it is well-known that the incident optical helicity appears from the determination of power emitted in the far zone. Because of this, we call reactive dichroism the phenomenon by which this incident reactive helicity becomes discriminatory for enantiomeric separation. We propose near field observation experiments of this reactive phenomenon.

Given the interplay between reactive and radiative quantities of electromagnetic fields, we believe that the concepts studied in this work enrich the landscape of photonics as regards nanoantennas and nanoparticle interactions with light. We expect that the observability of these reactive effects and quantities should form the basis of future experiments and techniques. In this respect, addressing reactive quantities on higher order multipole resonance excitation will be a subject of interest for future studies. This is of special interest for, e.g., nanosensing advances [76] and all-dielectric thermonanophotonics [77], to be used in effective biomedical diagnosis and therapies.

Although our analysis has been focused on the nanoscale, these results are equally valid in the microwave range due the scaling property of high index particles as Huygens sources, which remain with the same characteristics of generating large electric and magnetic dipole and multipole resonances as the illumination wavelength increases.

\section{ACKNOWLEDGMENTS}

M.N.-V. work was supported by Ministerio de Ciencia e Innovación of Spain, grant PGC2018-095777-B-C21. X.X. acknowledges the National Natural Science Foundation of China (11804119). Helpful comments from two anonymous referees are appreciated.

\section{APPENDIX A: PROOF OF EQS. (6) AND (7) FOR THE ENERGY FLOW} have

Since $\mathbf{B}(\mathbf{r})=(1 / i k) \nabla \times \mathbf{E}(\mathbf{r})$, using Eqs. (4) and (5), we

$$
\mathbf{B}(\mathbf{r})=\frac{1}{k} \int_{-\infty}^{\infty} d^{2} \mathbf{K}[\mathbf{k} \times \mathbf{e}(\mathbf{K})] \exp \left[i\left(\mathbf{K} \cdot \mathbf{R}+k_{z} z\right)\right] .
$$

Then the CPV flux across the plane $z=0$ is

$$
\begin{aligned}
\Phi^{\text {Poynt }} & =\frac{c}{8 \pi} \int_{-\infty}^{\infty} d^{2} \mathbf{R}\left[\mathbf{E}(\mathbf{r}) \times \mathbf{B}^{*}(\mathbf{r})\right] \cdot \hat{z} \\
& \left.=\frac{c}{8 \pi} \frac{1}{k} \int_{-\infty}^{\infty} d^{2} \mathbf{R} \int_{-\infty}^{\infty} d^{2} \mathbf{K} d^{2} \mathbf{K}^{\prime}\left[\mathbf{e}\left(\mathbf{K}^{\prime}\right) \times\left(\mathbf{k}^{*} \times \mathbf{e}^{*}(\mathbf{K})\right)\right] \cdot \hat{z} \exp \left[i\left(\mathbf{K}^{\prime}-\mathbf{K}\right) \cdot \mathbf{R}+\left(k_{z}^{\prime}-k_{z}^{*}\right) z\right]\right\}
\end{aligned}
$$

The $\mathbf{R}$ integral yields $\delta\left(\mathbf{K}^{\prime}-\mathbf{K}\right)=(1 / 2 \pi)^{2} \int_{-\infty}^{\infty} d^{2} \mathbf{R} \exp \left[i\left(\mathbf{K}^{\prime}-\mathbf{K}\right) \cdot \mathbf{R}\right]$, and subsequent integration in $\mathbf{K}^{\prime}$ yields for the righthand side of (A2):

$$
\begin{aligned}
\Phi^{\text {Poynt }} & =\frac{\pi c}{2 k} \int_{\infty}^{\infty} d^{2} \mathbf{K}\left[\mathbf{k}^{*}|\mathbf{e}(\mathbf{K})|^{2}-\mathbf{e}^{*}(\mathbf{K})\left(\mathbf{k}^{*} \cdot \mathbf{e}(\mathbf{K})\right)\right] \cdot \hat{z} \\
& =\frac{\pi c}{2 k} \int_{K \leqslant k} d^{2} \mathbf{K} q_{h}\left|\mathbf{e}_{h}(\mathbf{K})\right|^{2}-i \frac{\pi c}{2 k} \int_{K>k} d^{2} \mathbf{K}\left[q_{e}\left|\mathbf{e}_{e}(\mathbf{K})\right|^{2}-2 q_{e}\left|e_{e z}(\mathbf{K})\right|^{2}\right],
\end{aligned}
$$

where the $\mathbf{K}$ integral is split into its homogeneous and evanescent parts with subscripts $h$ and $e$, respectively. Then $\mathbf{k}_{h}$ is the real wave vector $\left(\mathbf{K}, q_{h}\right)$ of the homogeneous propagating plane wave component of complex amplitude $\mathbf{e}_{h}(\mathbf{K})$, whereas the complex wave vector $\mathbf{k}_{e}=\left(\mathbf{K}, i q_{e}\right)$ corresponds to each evanescent plane wave component of complex amplitude $\mathbf{e}_{h}(\mathbf{K})$. Also we have taken into account that $\mathbf{k}_{h}^{*} \cdot \mathbf{e}_{h}(\mathbf{K})=0$ and $\mathbf{k}_{e} \cdot \mathbf{e}_{e}(\mathbf{K})=0$, therefore $\mathbf{k}_{e}^{*} \cdot \mathbf{e}_{e}(\mathbf{K})=2 \mathbf{K} \cdot \mathbf{e}_{e, \perp}(\mathbf{K})=-2 i q_{e} e_{e z}(\mathbf{K})$ and $\left[\mathbf{e}_{e}(\mathbf{K})=\left(\mathbf{e}_{e \perp}(\mathbf{K}), e_{e z}(\mathbf{K})\right]\right.$.

Taking the real and imaginary parts in (A3), one has

$$
\Phi^{\text {RPoynt }}=\frac{\pi c}{2 k} \int_{K \leqslant k} d^{2} \mathbf{K} q_{h}\left|\mathbf{e}_{h}(\mathbf{K})\right|^{2}
$$

and

$$
\Phi^{\mathrm{IPoynt}}=-\frac{\pi c}{2 k} \int_{K>k} d^{2} \mathbf{K}\left[q_{e}\left|\mathbf{e}_{e}(\mathbf{K})\right|^{2}-2 q_{e}\left|e_{e z}(\mathbf{K})\right|^{2}\right]
$$

which are Eqs. (6) and (7) of the main text. 


\section{APPENDIX B: PROOF OF EQS. (17) AND (18)}

Using Eqs. (4) and (5), the integral on $z=0$ of $\mathbf{E}(\mathbf{r}) \cdot \mathbf{B}^{*}(\mathbf{r})$ is

$$
\left.\int_{-\infty}^{\infty} d^{2} \mathbf{R}\left[\mathbf{E}(\mathbf{r}) \cdot \mathbf{B}^{*}(\mathbf{r})\right]=\frac{1}{k} \int_{-\infty}^{\infty} d^{2} \mathbf{R} \int_{-\infty}^{\infty} d^{2} \mathbf{K} d^{2} \mathbf{K}^{\prime}\left[\mathbf{e}\left(\mathbf{K}^{\prime}\right) \cdot\left(\mathbf{k}^{*} \times \mathbf{e}^{*}(\mathbf{K})\right)\right] \exp \left[i\left(\mathbf{K}^{\prime}-\mathbf{K}\right) \cdot \mathbf{R}\right]\right\} .
$$

And following the same procedure as in Appendix A, we are led to

$$
\begin{aligned}
\int_{-\infty}^{\infty} d^{2} \mathbf{R}\left[\mathbf{E}(\mathbf{R}) \cdot \mathbf{B}^{*}(\mathbf{R})\right]= & \frac{(2 \pi)^{2}}{k} \int_{-\infty}^{\infty} d^{2} \mathbf{K} \mathbf{k}^{*} \cdot\left[\mathbf{e}^{*}(\mathbf{K}) \times \mathbf{e}(\mathbf{K})\right] \\
= & \frac{(2 \pi)^{2}}{k}\left\{\int_{K \leqslant k} d^{2} \mathbf{K} \mathbf{k}_{h} \cdot\left[\mathbf{e}_{h}^{*}(\mathbf{K}) \times \mathbf{e}_{h}(\mathbf{K})\right]+\int_{K>k} d^{2} \mathbf{K K} \cdot\left[\mathbf{e}_{e}^{*}(\mathbf{K}) \times \mathbf{e}_{e}(\mathbf{K})\right]\right. \\
& \left.+\int_{K>k} d^{2} \mathbf{K}\left(-i q_{e}\right)\left[\mathbf{e}_{e}^{*}(\mathbf{K}) \times \mathbf{e}(\mathbf{K})\right]_{z}\right\} .
\end{aligned}
$$

We recall that $\left[\mathbf{e}^{*}(\mathbf{K}) \times \mathbf{e}(\mathbf{K})\right]=i \Im\left[\mathbf{e}^{*}(\mathbf{K}) \times \mathbf{e}(\mathbf{K})\right]=(4 k i / c) \mathcal{F}_{E}(\mathbf{K})$; where $\mathcal{F}_{E}(\mathbf{K})$ is the density of electric spin of each angular plane wave component in $\mathbf{K}$ space. Then, taking the imaginary and real parts in (B2) and using the subindex $h$ and $e$ for homogeneous and evanescent components, we arrive at

$$
\begin{aligned}
\int_{-\infty}^{\infty} d^{2} \mathbf{R} \mathscr{H}(\mathbf{R}, 0) & =(1 / 2 k) \sqrt{\frac{\epsilon}{\mu}} \int_{-\infty}^{\infty} d^{2} \mathbf{R} \operatorname{Im}\left\{\mathbf{E}(\mathbf{R}) \cdot \mathbf{B}^{*}(\mathbf{R})\right\} \\
& =2 \frac{(2 \pi)^{2}}{k c}\left\{\int_{K \leqslant k} d^{2} \mathbf{K k}_{h} \cdot \mathcal{F}_{E h}(\mathbf{K})+\int_{K>k} d^{2} \mathbf{K K} \cdot \mathcal{F}_{E e \perp}(\mathbf{K})\right\}
\end{aligned}
$$

and

$$
\int_{-\infty}^{\infty} d^{2} \mathbf{R} \mathscr{H}_{\mathscr{R}}(\mathbf{R}, 0)=(1 / 2 k) \sqrt{\frac{\epsilon}{\mu}} \int_{-\infty}^{\infty} d^{2} \mathbf{R} \operatorname{Re}\left\{\mathbf{E}(\mathbf{R}) \cdot \mathbf{B}^{*}(\mathbf{R})\right\}=2 \frac{(2 \pi)^{2}}{k c} \int_{K>k} d^{2} \mathbf{K} q_{e} \mathcal{F}_{E e z}(\mathbf{K})
$$

In Eq. (B3), $\mathcal{F}_{E e}=\left(\mathcal{F}_{E e \perp}, \mathcal{F}_{E e z}\right), \mathcal{F}_{E e \perp}=\left(\mathcal{F}_{E e x}, \mathcal{F}_{E e y}\right)$. Equations (B3) and (B4) are (17) and (18) of the main text.

\section{APPENDIX C: EVANESCENT WAVE: TIME-AVERAGED QUANTITIES, ELECTRIC AND MAGNETIC IMAGINARY SPIN, AND ORBITAL MOMENTA}

The real part of $\mathbf{S}$ in (22) is

$$
\begin{aligned}
\operatorname{Re}\{\mathbf{S}\}= & \langle\mathbf{S}\rangle=\frac{c}{8 \pi \mu}\left[\frac{K}{k}\left(\left|T_{\perp}\right|^{2}+\left|T_{\|}\right|^{2}\right),\right. \\
& \left.2 \frac{K q}{k^{2}} \operatorname{Im}\left\{T_{\perp} T_{\|}^{*}\right\}, 0\right] \exp (-2 q z) \\
= & \frac{c k}{K}\left[w,-\frac{q}{4 \pi} \mathscr{H}, 0\right] .
\end{aligned}
$$

$\mathbf{S}$ is well-known to be associated to the optical force on a body. Considering a magnetodielectric dipolar particle placed in the air on the $z=0$ interface, the real part, or energy flow density $\langle\mathbf{S}\rangle$, of the CPV is known to constitute a momentum of the radiation whose $x$ component, proportional to $w$, gives rise to an $x$ force on the particle, separately acting on the particle electric (e) and magnetic (m) induced dipoles [21]. The component $\langle\mathbf{S}\rangle_{y}$ is known to produce a lateral force proportional to $-\mathscr{H}$ along $O Y$ [22], due to the interference of its e and $\mathrm{m}$ dipoles [21].

On the other hand, the time averages of the density of spin angular momentum, $\langle\mathcal{S}\rangle=\left(1 / 4 \pi c^{2}\right) \mathcal{F}=(1 / 2)\left(\left\langle\mathcal{S}_{e}\right\rangle+\right.$ $\left.\left\langle\mathcal{S}_{m}\right\rangle\right)$, where $\left\langle\mathcal{S}_{e}\right\rangle=(1 / 8 \pi k c) \mathfrak{\Im}\left\{\mathbf{E}^{*} \times \mathbf{E}\right\}$ and $\left\langle\mathcal{S}_{m}\right\rangle=$
$(1 / 8 \pi k c) \operatorname{Im}\left\{\mathbf{B}^{*} \times \mathbf{B}\right\}$, and of spin curl, or Belinfante spin momentum, $\left\langle\mathbf{P}^{S}\right\rangle=(1 / 2)\left[\left\langle\mathbf{P}_{e}^{S}\right\rangle+\left\langle\mathbf{P}_{m}^{S}\right\rangle\right]=(1 / 2) \nabla \times\langle\mathcal{S}\rangle$, are

$$
\begin{aligned}
\langle\mathcal{S}\rangle= & \frac{1}{8 \pi c}\left[\frac{-2 K}{k^{2}} \operatorname{Im}\left\{T_{\perp} T_{\|}^{*}\right\},-\frac{K q}{k^{3}}\left(\left|T_{\perp}\right|^{2}+\left|T_{\|}\right|^{2}\right), 0\right] \\
& \times \exp (-2 q z)=\frac{1}{4 \pi c K}\left[k \mathscr{H},-\frac{4 \pi q}{k} w, 0\right] .
\end{aligned}
$$

With $\left\langle\mathbf{P}_{e}^{S}\right\rangle=(1 / 2) \nabla \times\left\langle\mathcal{S}_{e}\right\rangle,\left\langle\mathbf{P}_{m}^{S}\right\rangle=(1 / 2) \nabla \times\left\langle\mathcal{S}_{m}\right\rangle$, and $\left\langle\mathbf{P}^{S}\right\rangle$ being:

$$
\begin{aligned}
\left\langle\mathbf{P}_{e}^{S}\right\rangle= & \frac{1}{8 \pi c}\left[-\frac{2 K q^{2}}{k^{3}}\left|T_{\|}\right|^{2} \frac{2 K q}{k^{2}} \operatorname{Im}\left\{T_{\perp} T_{\|}^{*}\right\}, 0\right] \\
& \times \exp (-2 q z) \\
\left\langle\mathbf{P}_{m}^{S}\right\rangle= & \frac{1}{8 \pi c}\left[-\frac{2 K q^{2}}{k^{3}}\left|T_{\perp}\right|^{2}, \frac{2 K q}{k^{2}} \operatorname{Im}\left\{T_{\perp} T_{\|}^{*}\right\}, 0\right] \\
& \times \exp (-2 q z), \\
\left\langle\mathbf{P}^{S}\right\rangle= & \frac{1}{8 \pi c}\left[-\frac{K q^{2}}{k^{3}}\left(\left|T_{\perp}\right|^{2}+\left|T_{\|}\right|^{2}\right) \frac{2 K q}{k^{2}} \Im\left\{T_{\perp} T_{\|}^{*}\right\}, 0\right] \\
& \times \exp (-2 q z)=-\frac{q}{c K}\left[\frac{q}{k} w, \frac{k}{4 \pi} \mathscr{H}, 0\right] .
\end{aligned}
$$

Equation (C2) remarks that, in agreement with the second term of the right-hand side of (17), $\mathscr{H}$ appears in the projection of the density of spin momentum $\langle\mathcal{S}\rangle$ on the $x$ direction, which is that of propagation of the evanescent wave $[78,79]$. On the other hand, (C5) highlights the transversal 
$y$ component of the spin momentum proportional to $\mathscr{H}$, as well as its longitudinal component, along $O X$, proportional to $w$.

Since the time-average electromagnetic field momentum density $\langle\mathbf{g}\rangle=\langle\mathbf{S}\rangle / c^{2}$ holds

$$
\langle\mathbf{g}\rangle=\left\langle\mathbf{P}^{O}\right\rangle+\left\langle\mathbf{P}^{S}\right\rangle ;
$$

$\left\langle\mathbf{P}^{O}\right\rangle$ being the density of time-averaged orbital momentum, one sees from $(\mathrm{C} 1)$ and $(\mathrm{C} 5)$ that the transverse $y$ component of $\langle\mathbf{g}\rangle$ comes from $\left\langle P_{y}^{S}\right\rangle$, which is characterized by $\mathscr{H}$. Both $\left\langle g_{y}\right\rangle$ and $\left\langle P_{y}^{S}\right\rangle$ are $(-1 / 4 \pi c)(k q / K) \mathscr{H} \exp (-2 q z)$. Therefore the transverse $y$ component $\left\langle g_{y}\right\rangle$ of the field momentum is provided by the transverse $y$ component $\left\langle P_{y}^{S}\right\rangle$ of Belinfante's momentum, in agreement with Ref. [22].

In addition, from (C6), (C5) and (C1) we have for the $i$ th Cartesian component of the time-averaged orbital momentum density,

$$
\begin{aligned}
\left\langle P_{i}^{O}\right\rangle= & (1 / 2)\left(\left\langle P_{e i}^{O}\right\rangle+\left\langle P_{m i}^{O}\right\rangle\right) \\
= & (1 / 2)(1 / 8 \pi k c)\left[\operatorname{Im}\left\{E_{j}^{*} \partial_{i} E_{j}\right\}+\operatorname{Im}\left\{B_{j}^{*} \partial_{i} B_{j}\right\}\right], \\
& (i, j=x, y, z), \\
\left\langle\mathbf{P}^{O}\right\rangle= & \frac{1}{8 \pi c}\left[\frac{K^{3}}{k^{3}}\left(\left|T_{\perp}\right|^{2}+\left|T_{\|}\right|^{2}\right), 0,0\right] \exp (-2 q z) \\
= & \frac{w}{c}\left[\frac{K}{k}, 0,0\right] .
\end{aligned}
$$

With the electric and magnetic orbital momenta:

$$
\begin{aligned}
\left\langle\mathbf{P}_{e}^{O}\right\rangle= & \frac{1}{8 \pi c}\left[\frac{K\left(2 K^{2}-k^{2}\right)}{k^{3}}\left|T_{\|}\right|^{2}+\frac{K}{k}\left|T_{\perp}\right|^{2}, 0,0\right] \\
& \times \exp (-2 q z)
\end{aligned}
$$

and

$$
\begin{aligned}
\left\langle\mathbf{P}_{m}^{O}\right\rangle= & \frac{1}{8 \pi c}\left[\frac{K\left(2 K^{2}-k^{2}\right)}{k^{3}}\left|T_{\perp}\right|^{2}+\frac{K}{k}\left|T_{\|}\right|^{2}, 0,0\right] \\
& \times \exp (-2 q z),
\end{aligned}
$$

respectively. $\left\langle\mathbf{P}^{O}\right\rangle$ contains the energy density $w$ of the wave and, as such, points in the propagation direction $O X$ of the wave, like both $\left\langle g_{x}\right\rangle$ and the $x$ component, $K>k$, of its propagation wave vector. This, in agreement with Ref. [22], confers to the evanescent wave a superluminal group velocity; although of course the time-average energy flow $\left\langle S_{x}\right\rangle$ propagates with speed less than $c$. Hence $\left\langle P_{x}^{O}\right\rangle$ pushes the aforementioned small particle, placed in the air on the interface, as radiation pressure along the $x$-propagation direction.

On the other hand, the electric and magnetic imaginary momenta of (25) are

$$
\begin{aligned}
\left(\mathbf{P}_{e}^{S I}\right)_{i} & =\frac{1}{8 \pi k c} \operatorname{Re}\left\{\partial_{j}\left(E_{i}^{*} E_{j}\right)\right\}, \\
\left(\mathbf{P}_{m}^{S I}\right)_{i} & =\frac{1}{8 \pi k c} \operatorname{Re}\left\{\partial_{j}\left(B_{i}^{*} B_{j}\right)\right\}, \\
\left(\mathbf{P}_{e}^{O I}\right)_{i} & =-\frac{1}{8 \pi k c} \partial_{j} \frac{1}{2} \delta_{i j}|\mathbf{E}|^{2}=-\frac{1}{8 \pi k c} \frac{1}{2} \partial_{i}|\mathbf{E}|^{2} \\
\left(\mathbf{P}_{m}^{O I}\right)_{i} & =-\frac{1}{8 \pi k c} \frac{1}{2} \partial_{i}|\mathbf{B}|^{2}(i, j=x, y, z), \\
\mathbf{P}_{e}^{S I} & =\frac{1}{4 \pi k^{2} c}\left(0,-K q \operatorname{Re}\left\{T_{\perp} T_{\|}^{*}\right\},-\frac{K^{2} q}{k}\left|T_{\|}\right|^{2}\right) \exp (-2 q z), \\
\mathbf{P}_{m}^{S I} & =\frac{1}{4 \pi k^{2} c}\left(0, K q \operatorname{Re}\left\{T_{\perp} T_{\|}^{*}\right\},-\frac{K^{2} q}{k}\left|T_{\perp}\right|^{2}\right) \exp (-2 q z),
\end{aligned}
$$

$$
\mathbf{P}_{e}^{O I}=\frac{q}{8 \pi k c}\left(0,0, \frac{K^{2}+q^{2}}{k^{2}}\left|T_{\|}\right|^{2}+\left|T_{\perp}\right|^{2}\right) \exp (-2 q z)
$$

$$
\mathbf{P}_{m}^{O I}=\frac{q}{8 \pi k c}\left(0,0, \frac{K^{2}+q^{2}}{k^{2}}\left|T_{\perp}\right|^{2}+\left|T_{\|}\right|^{2}\right) \exp (-2 q z) \text {. }
$$

\section{APPENDIX D: DIPOLE FIELDS}

We express the fields emitted by a dipole with electric and magnetic moments $\mathbf{p}$ and $\mathbf{m}$ as [9]

$$
\begin{gathered}
\mathbf{E}^{(s)}(\mathbf{r})=\left\{\frac{k^{2}}{\epsilon r}[\hat{\boldsymbol{r}} \times(\mathbf{p} \times \hat{\boldsymbol{r}})]+\frac{1}{\epsilon}[3 \hat{\boldsymbol{r}}(\hat{\boldsymbol{r}} \cdot \mathbf{p})-\mathbf{p}]\left(\frac{1}{r^{3}}-\frac{i k}{r^{2}}\right)-\sqrt{\frac{\mu}{\epsilon}}(\hat{\boldsymbol{r}} \times \mathbf{m})\left(\frac{k^{2}}{r}+\frac{i k}{r^{2}}\right)\right\} e^{i k r}(\mathbf{r}=r \hat{\boldsymbol{r}}), \\
\mathbf{B}^{(s)}(\mathbf{r})=\left\{\frac{\mu k^{2}}{r}[\hat{\boldsymbol{r}} \times(\mathbf{m} \times \hat{\boldsymbol{r}})]+\mu[3 \hat{\boldsymbol{r}}(\hat{\boldsymbol{r}} \cdot \mathbf{m})-\mathbf{m}]\left(\frac{1}{r^{3}}-\frac{i k}{r^{2}}\right)+\sqrt{\frac{\mu}{\epsilon}}(\hat{\boldsymbol{r}} \times \mathbf{p})\left(\frac{k^{2}}{r}+\frac{i k}{r^{2}}\right) e^{i k r} .\right.
\end{gathered}
$$

\section{APPENDIX E: PROOF OF EQS. (34) AND (35)}

The reactive power $W_{\text {react }}^{(s)}=\frac{c k}{3 r^{3}}\left(|\mathbf{p}|^{2}-|\mathbf{m}|^{2}\right)$ may also be obtained by performing the volume integration of the right-hand side of (34) for the magnetoelectric dipole:

$$
\left\langle\tilde{W}_{e}^{(s)}\right\rangle=\int_{V_{\infty}-V} d^{3} r\left\langle\tilde{w}_{e}^{(s)}\right\rangle=\frac{1}{16 \pi} \int_{r}^{\infty} d r r^{2} \int_{0}^{2 \pi} d \phi \int_{0}^{\pi} d \theta \sin \theta\left[\left|\mathbf{E}^{(s)}\right|^{2}-\left|\mathbf{E}_{\mathrm{FF}}^{(s)}\right|^{2}\right]=\frac{k^{3}}{6}\left[|\mathbf{p}|^{2}\left(\frac{1}{(k r)^{3}}+\frac{1}{k r}\right)+\frac{|\mathbf{m}|^{2}}{k r}\right]
$$


and

$$
\left\langle\tilde{W}_{m}^{(s)}\right\rangle=\int_{V_{\infty}-V} d^{3} r\left\langle\tilde{w}_{m}^{(s)}\right\rangle=\frac{1}{16 \pi} \int_{r}^{\infty} d r r^{2} \int_{0}^{2 \pi} d \phi \int_{0}^{\pi} d \theta \sin \theta\left[\left|\mathbf{B}^{(s)}\right|^{2}-\left|\mathbf{B}_{\mathrm{FF}}^{(s)}\right|^{2}\right]=\frac{k^{3}}{6}\left[|\mathbf{m}|^{2}\left(\frac{1}{(k r)^{3}}+\frac{1}{k r}\right)+\frac{|\mathbf{p}|^{2}}{k r}\right] .
$$

Having used Eqs. (D1) and (D2) of Appendix D, and $\mathbf{E}_{\mathrm{FF}}^{(s)}$ and $\mathbf{B}_{\mathrm{FF}}^{(s)}$ being the radiated far fields with $r^{-1}$ dependence. Thus the time-averaged reactive power is

$$
2 \omega\left(\left\langle\tilde{W}_{m}^{(s)}\right\rangle-\left\langle\tilde{W}_{e}^{(s)}\right\rangle\right)=W_{\text {react }}^{(s)}=\frac{c k}{3 r^{3}}\left(|\mathbf{m}|^{2}-|\mathbf{p}|^{2}\right)
$$

which is (34).

The total time-averaged energy outside the volume $V$ is after Eqs. (E1) and (E2),

$$
\left\langle\tilde{W}_{T}^{(s)}\right\rangle=\left\langle\tilde{W}_{e}^{(s)}\right\rangle+\left\langle\tilde{W}_{m}^{(s)}\right\rangle=\frac{1}{6}\left(|\mathbf{p}|^{2}+|\mathbf{m}|^{2}\right)\left(\frac{1}{r^{3}}+\frac{2 k^{2}}{r}\right),
$$

which is (35) when $r \rightarrow a$.

[1] H. Raether, Surface Plasmons on Smooth and Rough Surfaces and on Gratings (Springer-Verlag, Berlin, 1988).

[2] A. A. Kolokolov and G. V. Skrotskii, Interference of reactive components of an electromagnetic field, Sov. Phys. Usp. 35, 1089 (1992).

[3] R. F. Harrington, Time-harmonic Electromagnetic Fields (J. Wiley, New York, 2001).

[4] Optics at the Nanometer Scale: Imaging and Storing with Photonic near Fields, edited by M. Nieto-Vesperinas and N. Garcia (Kluwer, Dordrecht, 1996).

[5] J. A. Stratton, Electromagnetic Theory (Mc Graw-Hill, New York, 1941).

[6] H. A. Wheeler, Fundamental limitations of small antennas, Proc. IRE 35, 1479 (1947).

[7] L. J. Chu, Physical limitations on omni-directional antennas, J. Appl. Phys. 19, 1163 (1948).

[8] R. E. Collin and S. Rothschild, Evaluation of antenna $Q$, IEEE Trans. Antennas Propagat. AP-12, 23 (1964); R. E. Collin, Minimum $Q$ of small Antennas, J. Electromag. Waves Appl. 121369 (1998).

[9] J. D. Jackson, Classical Electrodynamics, 2nd ed. (Wiley, New York, 1975).

[10] J. S. McLean, A Re-examination of the fundamental limits on the radiation $\mathrm{q}$ of electrically small antennas, IEEE Trans. Antennas Propag. AP-44, 672 (1996).

[11] W. Geyi and P. Jarmuszewski, The foster reactance theorem for antennas and radiation Q, IEEE Trans. Antenn. Propag. 48, 401 (2000); W. Geyi, Foundatios of Applied Electrodynamics (J. Wiley, New York, 2010). Sec. 4.4.1.

[12] C. A. Balanis, Antenna Theory, 4th ed. (Wiley, New York, 2016).

[13] C. A. Valagiannopoulos and A. Alu, The role of reactive energy in the radiation by a dipole antenna, IEEE Trans. Antennas Propag. 63, 3736 (2015).

[14] Y. Tang and A. E. Cohen, Optical Chirality and Its Interaction with Matter, Phys. Rev. Lett. 104, 163901 (2010).

[15] R. P. Cameron, S. M. Barnett and A. M. Yao, Optical helicity, optical spin and related quantities in electromagnetic theory, New. J. Phys. 14, 053050 (2012).
[16] I. Fernandez-Corbaton and G. Molina-Terriza, Role of duality symmetry in transformation optics, Phys. Rev. B 88, 085111 (2013).

[17] M. Nieto-Vesperinas, Optical theorem for the conservation of electromagnetic helicity: significance for molecular energy transfer and enantiomeric discrimination by circular dichroism, Phys. Rev. A 92, 023813 (2015); Chiral optical fields: a unified formulation of helicity scattered from particles and dichroism enhancement, Philos. Trans. R. Soc. A 375, 20160314 (2017).

[18] S. Nechayev and P. Banzer, Mimicking chiral light-matter interaction, Phys. Rev. B 99, 241101(R) (2019).

[19] F. Crimin, N. Mackinnon, J. B. Götte, and S. M. Barnett, Optical helicity and chirality: Conservation and sources, Appl. Sci. 9, 828 (2019).

[20] S. Yan, M. Li, Y. Liang, Y. Cai and B. Yao, Spin momentumdependent orbital motion, New J. Phys. 22, 053009 (2020).

[21] M. Nieto-Vesperinas, J. J. Saenz, R. Gomez-Medina, and L. Chantada, Optical forces on small magnetodielectric particles, Opt. Express 18, 11428 (2010).

[22] K. Y. Bliokh, A. Y. Bekshaev and F. Nori, Extraordinary momentum and spin in evanescent waves, Nat. Commun. 5, 3300 (2014); M. Antognozzi, C. R. Bermingham, R. L. Harniman, S. Simpson, J. Senior, R. Hayward, H. Hoerber, M. R. Dennis, A. Y. Bekshaev, K. Y. Bliokh, and F. Nori, Direct measurements of the extraordinary optical momentum and transverse spindependent force using a nano-cantilever, Nat. Phys. 12, 731 (2016).

[23] K. Y. Bliokh and F. Nori, Transverse and longitudinal angular momenta of light, Phys. Rep. 592, 1 (2015).

[24] K. Y. Bliokh, Y. S. Kivshar, and F. Nori, Magnetoelectric Effects in Local Light-Matter Interactions, Phys. Rev. Lett. 113, 033601 (2014).

[25] X. Xu and M. NietoVesperinas, Azimuthal Imaginary Poynting Momentum Density, Phys. Rev. Lett. 123, 233902 (2019).

[26] X. Xu, M. Nieto-Vesperinas, C.-W. Qiu, X. Liu, D. Gao, Y. Zhang, and B. Li, Kerker-type intensity-gradient force of light, Laser Photonics Rev. 14, 1900265 (2020).

[27] P. Bharadwaj, B. Deutsch, and L. Novotny, Optical antennas, Adv. Opt. Photon. 1, 438 (2009); 
L. Novotny and N. van Hulst, Antennas for light, Nat. Photon. 5, 83 (2011).

[28] L. V. Poulikakos, P. Thureja, A. Stollmann, E. De Leo, and D. J. Norris, Chiral light design and detection inspired by optical antenna theory, Nano Lett. 18, 4633 (2018).

[29] W. L. Barnes, S. A. R. Horsley, and W. L. Vos, Classical antennas, quantum emitters, and densities of optical states, J. Opt. 22, 073501 (2020).

[30] P. Biagioni, J.-S. Huang and B. Hecht, Nanoantennas for visible and infrared radiation, Rep. Prog. Phys. 75, 024402 (2012).

[31] I. Liberal, I. Ederra, R. Gonzalo and R. W. Ziolkowski, Induction Theorem Analysis of Resonant Nanoparticles: Design of a Huygens Source Nanoparticle Laser, Phys. Rev. Appl. 1, 044002 (2014).

[32] I. Liberal and N. Engheta, Nonradiating and radiating modes excited by quantum emitters in open epsilon-near-zero cavities, Sci. Adv. 2, e1600987 (2016).

[33] M. S. Bin-Alam, O. Reshef, Y. Mamchur, M. Z. Alam, G. Carlow, J. Upham, B. T. Sullivan, J.-M. Ménard, M. J. Huttunen, R. W. Boyd, and K. Dolgaleva, Ultra-high-Q resonances in plasmonic metasurfaces, Nat. Commun. 12, 974 (2021).

[34] R. Won, Into the Mie-tronic era, Nat. Photon. 13, 585 (2019).

[35] N. Bonod and Y. Kivshar, All-dielectric Mie-resonant metaphotonics, Compt. Rendus Phys. 21, 425 (2020).

[36] A. Garcia-Etxarri, R. Gomez-Medina, L. S. Froufe-Perez, C. Lopez, L. Chantada, F. Scheffold, J. Aizpurua, M. NietoVesperinas and J. J. Saenz, Strong magnetic response of submicron Silicon particles in the infrared, Opt. Express 19, 4816 (2011).

[37] M. Nieto-Vesperinas, R. Gomez-Medina and J. J. Saenz, Angle suppressed scattering and optical forces on submicrometer dielectric particles, J. Opt. Soc. Am. A 28, 54 (2011).

[38] A. I. Kuznetsov, A. E. Miroshnichenko, Y. H. Fu, J. B. Zhang and B. Luk'yanchuk, Magnetic light, Sci. Rep. 2, 492 (2012).

[39] M. Decker and I. Staude, Resonant dielectric nanostructures: a low-loss platform for functional nanophotonics, J. Opt. 18, 103001 (2016); I. Staude, T. Pertsch and Y. S. Kivshar, Alldielectric resonant Meta-Optics lightens up, ACS Photonics 6, 802 (2019).

[40] A. I. Kuznetsov, A. E. Miroshnichenko, M. L. Brongersma, Y. S. Kivshar, B. Luk'yanchuk, Optically resonant dielectric nanostructures, Science 354, 2472 (2016).

[41] M. Nieto-Vesperinas, Fundamentals of Mie scattering, in Dielectric Metamaterials: Fundamentals, Designs, and Applications, edited by I. Brener, S. Liu, I. Staude, J. Valentine, and C. Holloway (Elsevier, Amsterdam, 2019), Chap. 2; R. Paniagua-Dominguez, B. Luk'yanchuk, and A. I. Kuznetsov, Control of scattering by isolated dielectric nanoantennas, in Dielectric Metamaterials: Fundamentals, Designs, and Applications, edited by I. Brener, S. Liu, I. Staude, J. Valentine, and C. Holloway (Elsevier, Amsterdam, 2019), Chap. 3.

[42] The helicity of time-harmonic wavefields is proportional to their chirality [14]. Both quantities differ only by the square of the wave number [17].

[43] E. O. Kamenetskii, M. Berezin, and R. Shavit, Microwave magnetoelectric fields: helicities and reactive power flows, Appl. Phys. B 121, 31 (2015).
[44] M. Kerker, D. S. Wang, and C. L. Giles, Electromagnetic scattering by magnetic spheres, J. Opt. Soc. Am. 73, 765 (1983).

[45] R. Gomez-Medina, B. Garcia-Camara, I. Suarez-Lacalle, F. Gonzalez, F. Moreno, M. Nieto-Vesperinas and J. J. Saenz, Electric and magnetic dipolar response of germanium nanospheres: interference effects, scattering anisotropy, and optical forces, J. Nanophotonics 5, 053512 (2011).

[46] J. M. Geffrin, B. Garcia-Camara, R. Gomez-Medina, P. Albella, L. S. Froufe-Perez, C. Eyraud, A. Litman, R. Vaillon, F. Gonzalez, M. Nieto-Vesperinas, J. J. Saenz, and F. Moreno, Magnetic and electric coherence in forward- and back-scattered electromagnetic waves by a single dielectric subwavelength sphere, Nat. Commun. 3, 1171 (2012).

[47] S. Person, M. Jain, Z. Lapin, J. J. Saenz, G. Wicks and L. Novotny, Demonstration of zero optical backscattering from single nanoparticles, Nano Lett. 13, 1806 (2013).

[48] A. Bag, M. Neugebauer, P. Woźniak, G. Leuchs, and P. Banzer, Transverse Kerker Scattering for Angstrom Localization of Nanoparticles, Phys. Rev. Lett. 121, 193902 (2018).

[49] J. Olmos-Trigo, C. Sanz-Fernandez, D. R. Abujetas, J. LasaAlonso, N. de Sousa, A. García-Etxarri, J. A. Sanchez-Gil, G. Molina-Terriza and J. J. Saenz, Kerker Conditions upon Lossless, Absorption, and Optical Gain Regimes, Phys. Rev. Lett. 125, 073205 (2020); J. Olmos-Trigo, D. R. Abujetas, C. Sanz-Fernández, J. A. Sánchez-Gil, and J. J. Saenz, Optimal backward light scattering by dipolar particles, Phys. Rev. Research 2, 013225 (2020); J. Olmos-Trigo, D. R. Abujetas, C. Sanz-Fernandez, X. Zambrana-Puyalto, N. de Sousa, J. A. Sanchez-Gil, and J. J. Saenz, Unveiling dipolar spectral regimes of large dielectric Mie spheres from helicity conservation, Phys. Rev. Res. 2, 043021 (2020).

[50] If the embedding medium is lossy and hence it also presents a certain dispersion, or complex, there will be terms associated with effects additional to those adressed in this research. See, e.g., Sec. 1.8 of Ref. [3] for the complex Poynting theorem and Ref. [54] for the conservation of optical chiraliity. For instance, for lossy dispersive media the electric and magnetic energy terms should be of the form $(1 / 16 \pi) \partial_{\omega}\left\{\omega\left[\epsilon^{\prime}(\omega)+i \epsilon^{\prime \prime}(\omega)\right]\right\}|\mathbf{E}|^{2}$ and $(1 / 16 \pi) \partial_{\omega}\left\{\omega\left[\mu^{\prime}(\omega)+i \mu^{\prime \prime}(\omega)\right]\right\}|\mathbf{H}|^{2}$, [see, e.g., Sec. 80 of L. D. Landau and E. M. Lifshitz, Electrodynamics of Continuous Media (Pergamon Press, Oxford, 1984)], which is in fact an approximation for time slowly-varying fields. Such generalizations, and their related problems [11,31] are outside the aims of this work.

[51] L. Mandel and E. Wolf, Optical Coherence and Quantum Optics Cambridge University Press, Cambridge, 1995).

[52] M. Nieto-Vesperinas, Scattering and Diffraction in Physical Optics, 2nd ed. (World Scientific, Singapore, 2006).

[53] K. Y. Bliokh, A. Y. Bekshaev and F. Nori, Dual electromagnetism: helicity, spin, momentum and angular momentum, New J. Phys. 15, 033026 (2013).

[54] L. V. Poulikakos, P. Gutsche, K. M. McPeak, S. Burger, J. Niegemann, C. Hafner, and D. J. Norris, Optical chirality flux as a useful far field probe of chiral near fields, ACS Photonics 3, 1619 (2016).

[55] P. Gutsche, L. V. Poulikakos, M. Hammerschmidt, S. Burger, and F. Schmidt, Time-harmonic optical chirality in inhomogeneous space, Proc. SPIE 9756, 97560X (2016). 
[56] P. Gutsche and M. Nieto-Vesperinas, Optical chirality of timeharmonic wavefields for classification of scatterers, Sci. Rep. 8, 9416 (2018).

[57] Notice the difference of Eqs. (14-(16) with the conservation equation and quantities of Ref. [54], (cf. Eqs. (S. I.12)-(S. I.15) of the Supporting Information of Ref. [54]). In homogeneous lossless media addressed here, (S. I.12)-(S. I.15) describe purely real quantities and $\chi_{e}-\chi_{m}=0$, so that the conservation equation (S. I.12) of Ref. [54] does not provide a law for reactive quantities, although both the optical chirality flux (S. I.15) and the real conservation equation (S. I.12) are $k^{2}$ times the flow of helicity (13) and the conservation of helicity (12), respectively. In order to get complex expressions (S. I.12)-(S. I.15), and $\left.\operatorname{Re}\left[\chi_{e}-\chi_{m}\right] \neq 0, \operatorname{Im}\left[\chi_{e}-\chi_{m}\right] \neq 0\right)$, lossy media should be considered, but then the imaginary parts of (S. I.12)-(S. I.15) are different to (14)-(16). Hence, the imaginary part (14) of the complex conservation equation (16) is a novel law for the reactive helicity $\mathscr{H}_{\mathscr{R}}$ and its flow $\mathcal{F}_{\mathscr{H}_{\mathscr{R}}}$.

[58] M. Nieto-Vesperinas, Optical torque: Electromagnetic spin and orbital-angular-momentum conservation laws and their significance, Phys. Rev. A 92, 043843 (2015).

[59] Let $\mathbf{E}=\left(e^{+} \boldsymbol{\epsilon}^{+}+e^{-} \boldsymbol{\epsilon}^{-}\right) \cos k z, \mathbf{B}=\left(e^{+} \boldsymbol{\epsilon}^{+}-e^{-} \boldsymbol{\epsilon}^{-}\right) \sin k z$, be an elliptically polarized standing wavefield, expressed in the helicity basis $\hat{\boldsymbol{\epsilon}}^{ \pm}=\frac{1}{\sqrt{2}}(\hat{\boldsymbol{x}} \pm i \hat{\boldsymbol{y}})$, with amplitudes $\operatorname{CPL}(+)$ (left circularly polarized) $e^{+}$and $\mathrm{CPL}(-)$ (right circularly polarized) $e^{-}$. One has $\mathbf{E} \times \mathbf{B}^{*}=-(i / 2)\left(\mid\left(\left.e^{+}\right|^{2}+\right.\right.$ $\left.\left|e^{-}\right|^{2}\right) \sin 2 k z \hat{z}, \quad|\mathbf{B}|^{2}-|\mathbf{E}|^{2}=-\left(\mid\left(\left.e^{+}\right|^{2}+\left|e^{-}\right|^{2}\right) \cos 2 k z, \quad\right.$ and hence $\nabla \cdot \Im\{\mathbf{S}\}=2 \omega\left(\left\langle w_{m}\right\rangle-\left\langle w_{e}\right\rangle\right)$. In addition, $\mathbf{E} \cdot \mathbf{B}^{*}=$ $(1 / 2)\left(\mid\left(\left.e^{+}\right|^{2}-\left|e^{-}\right|^{2}\right) \sin 2 k z, \mathbf{B}^{*} \times \mathbf{B}-\mathbf{E}^{*} \times \mathbf{E}=-i\left(\mid\left(\left.e^{+}\right|^{2}-\right.\right.\right.$ $\left.\left|e^{-}\right|^{2}\right) \cos 2 k z \hat{\boldsymbol{z}}$, so that $\nabla \cdot \mathcal{F}_{\mathscr{H}_{\mathscr{R}}}=2 \omega \mathscr{H}_{\mathscr{R}}$. Therefore this kind of wave has no time-averaged energy transport $\langle\mathbf{S}\rangle$, nor helicity density $\mathscr{H}$, but their densities of reactive power $2 \omega\left(\left\langle w_{m}\right\rangle-\right.$ $\left.\left\langle w_{e}\right\rangle\right)$, IPV, reactive helicity $\mathscr{H}_{\mathscr{R}}$ and its flow $\mathcal{F}_{\mathscr{H}_{\mathscr{R}}}$, are nonzero. Note that if the standing wave is linearly polarized, (e.g., $E_{y}=B_{x}=0$ ), it has IPV and stores reactive power, but $\mathscr{H}_{\mathscr{R}}=0$.

[60] M. Born and E. Wolf, Principles of Optics (Cambridge University Press, Cambridge 1995).

[61] M. Nieto-Vesperinas and J. J. Saenz, Optical forces from an evanescent wave on a magnetodielectric small particle, Opt. Lett. 35, 4078 (2010).

[62] P. C. Chaumet and M. Nieto-Vesperinas, Coupled dipole method determination of the electromagnetic force on a particle over a flat dielectric substrate, Phys. Rev. B 61, 14119 (2000).

[63] Note that $\left\langle\tilde{w}_{e}^{(s)}\right\rangle$ and $\left\langle\tilde{w}_{m}^{(s)}\right\rangle$ are not the electric and magnetic energy densities of only the near plus intermediate fields, say $\mathbf{E}^{(\mathrm{NF}, \mathrm{IF})}, \mathbf{B}^{(\mathrm{NF}, \mathrm{IF})}$, since they also contain an interference term of these fields with the far fields: $2 \operatorname{Re}\left\{\mathbf{E}^{(\mathrm{NF}, \mathrm{IF})} \cdot \mathbf{E}^{(\mathrm{FF})}\right\}$ and $2 \operatorname{Re}\left\{\mathbf{B}^{(\mathrm{NF},-\mathrm{F})} \cdot \mathbf{B}^{(\mathrm{FF})}\right\}$, respectively.

[64] C. F. Bohren and D. R. Huffman, Absorption and Scattering of Light by Small Particles (Wiley, New York, 1998).

[65] K. Koshelev and Y. Kivshar, Dielectric resonant metaphotonics, ACS Photon. 8, 102 (2021).

[66] M. V. Rybin, K. L. Koshelev, Z. F. Sadrieva, K. B. Samusev, A. A. Bogdanov, M. F. Limonov, and Y. S. Kivshar, High-Q Supercavity Modes in Subwavelength Dielectric Resonators, Phys Rev. Lett. 119, 243901 (2017).
[67] Note that one could address a medium composed of particles, in which transport of light occurs. The existence of multiple scattering may be associated with a slow convergence of the Born series due to some coupling between the particles, stringent conditions for convergence under excitation of their resonances, (see, e.g., N. A. Ustimenko, D. F. Kornovan, K. V. Baryshnikova, A. B. Evlyukhin, and M. I. Petrov, Multipole Born series approach to light scattering by Mie-resonant nanoparticle structures, arXiv:2108.11920v1) or even with no convergence at all if there is either strong coupling between the induced dipoles (or multipoles). The latter two effects should be associated with higher $Q$ factors, and thus with larger amounts of stored and reactive powers. The same we would expect to rule the behavior of the scattered helicity and a quality factor which might be introduced for this quantity; this being an area of possible future exploration.

[68] For a circularly polarized incident plane wave of unit amplitude, the induced dipoles are $\mathbf{p}=\alpha_{e} \mathbf{E}^{(i)}$ and $\mathbf{m}=\alpha_{m} \mathbf{B}^{(i)}$ with $\mathbf{E}^{(i)}=\hat{\boldsymbol{\epsilon}}^{ \pm}, \mathbf{B}^{(i)}=\mp i \hat{\boldsymbol{\epsilon}}^{ \pm}, \hat{\boldsymbol{\epsilon}}^{ \pm}=\frac{1}{\sqrt{2}}(\hat{\boldsymbol{x}} \pm i \hat{\boldsymbol{y}})$, the upper and lower sign applies according to whether it is left circular, CPL(+), or right circular, CPL(-), respectively. Since at K1: $\alpha_{m}=\alpha_{e}$, then $\mathbf{p}=\alpha_{e} \hat{\boldsymbol{\epsilon}}^{ \pm}$and $\mathbf{m}=\mp i \alpha_{e} \hat{\boldsymbol{\epsilon}}^{ \pm}$, and thus $\mathbf{p}= \pm i \mathbf{m}$ [17]. Therefore, at K1: $\mathbf{p} \cdot \mathbf{m}^{*}= \pm i\left|\alpha_{e}\right|^{2}$. However, at K2: $\alpha_{m}=-\alpha_{e}^{*}$ and hence $\mathbf{p} \cdot \mathbf{m}^{*}=\mp i \alpha_{e}^{2}$

[69] In this regard, we note that a reactive optical theorem in anisotropic media was reported by E. A. Marengo, A new theory of the generalized optical theorem in anisotropic media, IEEE Trans. Antenn. Propag. 61, 2164 (2013).

[70] In contrast with the energy OT and ROT, both helicity OT and ROT contain a real part in the extinction term. This is due to the different functional form in the real and imaginary parts, $\mathcal{F}$ and $\mathcal{F}_{\mathscr{H}_{\mathscr{R}}}$, of the complex flow of helicity $\mathcal{F}_{C}$, as seen in Eqs. (13)-(16). Equation (16) shows, at difference with those of the CPV.

[71] To prove that $\int_{\partial V_{\infty}} d^{2} r \mathcal{F}_{\mathscr{H}_{\mathscr{R}}}^{(s)} \cdot \mathbf{n}=0$, we write the scattered field at points in the far zone characterized by the direction unit vector $\mathbf{n}$ as: $\mathbf{E}^{(s)}(\mathbf{n})=\mathbf{e}(\mathbf{n}) \exp (i k r) / r$ and $\mathbf{H}^{(s)}(\mathbf{n})=$ $\mathbf{h}(\mathbf{n}) \exp (i k r) / r, \quad \mathbf{h}=\mathbf{n} \times \mathbf{e}$. Then it is straightforward to see that $\int_{\partial V_{\infty}} d^{2} r \operatorname{Im}\left\{\mathbf{h}^{*}(\mathbf{n}) \times \mathbf{h}(\mathbf{n})\right\} \cdot \mathbf{n}=\int_{\partial V_{\infty}} d^{2} r \operatorname{Im}\left\{\mathbf{e}^{*}(\mathbf{n}) \times\right.$ $\mathbf{e}(\mathbf{n})\} \cdot \mathbf{n}$. This latter equality and the definition (15) of $\mathcal{F}_{\mathscr{H}_{\mathscr{R}}}^{(s)}$ constitute the proof.

[72] L. D. Barron, Molecular Light Scattering and Optical Activity (Cambridge University Press, Cambridge, 2004).

[73] J. A. Schellman, Circular dichroism and optical rotation, Chem. Rev. 75, 323 (1975).

[74] In this connection, it should be noted that an expression akin to such dissymmetry factor, was written in [24] as what the authors called relative magnetoelectric response of the particle to the illumination. This was done on employing what they named magnetoelectric absorption rate, determined from $\Re\left[\mathbf{E}^{(i)} \cdot \mathbf{B}^{(i) *}\right]$, which they called magnetoelectric energy; even though the physical process that produces it was not reported. Here we have demonstrated that the left-hand side of Eqs. (54) and (51), as well as (52), describe the mechanism through which this quantity appears and may be observed.

[75] M. F. Picardi, A. V. Zayats, and F. J. Rodríguez-Fortuño, Janus and Huygens Dipoles: Near-Field Directionality Beyond Spin-Momentum Locking, Phys. Rev. Lett. 120, 117402 (2018). 
[76] A. Krasnok, M. Caldarola, N. Bonod, and A. Alú, Spectroscopy and Biosensing with Optically Resonant Dielectric Nanostructures, Adv. Opt. Mater. 6, 1701094 (2018).

[77] G. P. Zograf, M. I. Petrov, S. V. Makarov, and Y. S. Kivshar, Alldielectric thermonanophotonics, Adv. Opt. Photonics 13, 643 (2021).
[78] M. Neugebauer, T. Bauer, A. Aiello, and P. Banzer, Measuring the Transverse Spin Density of Light, Phys. Rev. Lett. 114, 063901 (2015).

[79] Notice that Eqs. (19) correspond to a wavefield $\mathbf{E}(\mathbf{r})$ with angular spectrum $\mathbf{e}\left(\mathbf{K}^{\prime}\right)=\left(-\frac{k_{z}^{\prime}}{k} T_{\|}, T_{\perp}, \frac{K^{\prime}}{k} T_{\|}\right) \delta\left(\mathbf{K}^{\prime}-\mathbf{K}\right),(\mathbf{k}=$ $\left.\left(\mathbf{K}, k_{z}\right), \mathbf{K}=\left(K_{x}, 0\right), k_{z}=i q, K_{x}>k\right)$. 